\title{
Optical colour maps of Seyfert galaxies
}

\author{
J.K. Kotilainen ${ }^{1,2}$ and M.J. Ward ${ }^{3, \star}$ \\ 1 Tuorla Observatory, University of Turku, Väisäläntie 20, FIN-21500 Piikkiö, Finland \\ 2 International School for Advanced Studies (ISAS/SISSA), via Beirut 2-4, I-34014 Trieste, Italy \\ e-mail: jkotilai@sissa.it \\ 3 Astrophysics, Nuclear Physics Building, Keble Road, Oxford OX1 3RH, UK \\ e-mail: m.ward1@physics.oxford.ac.uk
}

Received January 24; accepted May 20, 1996

\begin{abstract}
We present optical broad band colour maps of nine Seyfert galaxies. In the bands used, the contribution from emission lines to the total flux is relatively small, and hence the images predominantly trace the continuum distribution. The ratios of these images are sensitive to gradients in the stellar populations, and to reddening by dust. We detect ringlike, clumpy or elongated morphological features in several galaxies, which are blue in the $B-I$ index ( $V-I$ for NGC 7469). For NGC 7469 there is good spatial agreement with the molecular millimeter $\mathrm{CO}$ and radio continuum structure indicating the presence of star forming regions around its Seyfert nucleus. The blue elongations and/or double structures seen in the colour maps of NGC 1068, NGC 3227, NGC 4151, Mrk 3 and Mrk 573 are roughly parallel to their extended radio and NLR emission and probably arise from scattering of nuclear light by extranuclear "mirrors". In Mrk 78 and Mrk 348, the structure is not well resolved, possibly because the scattering mirrors are either less efficient and/or further away from the nucleus than in the previous galaxies. In NGC 1667, we detect double red maxima, again possibly related to obscuring material. The colours of the blue maxima are not well determined due to contamination from the underlying bulge, but they are generally consistent with those expected from scattering off dust or electrons. Our findings thus give further support to the current unified models of AGN.
\end{abstract}

Key words: galaxies: nuclei — galaxies: Seyfert galaxies: stellar content - galaxies: structure galaxies: photometry

Send offprint requests to: J.K. Kotilainen (SISSA address)

* Present address: Department of Physics and Astronomy, University of Leicester, University Road, Leicester LE1 7RH, UK

\section{Introduction}

An important aim of active galactic nuclei (AGN) research is to quantify the relative contributions of nonstellar and stellar (in some cases starburst) components within the nuclear regions. Based on emission line ratios in the extended narrow line region (ENLR), radio morphologies, and infrared (IR) images, it is known that some Seyfert nuclei have circumnuclear starburst regions (e.g. NGC 1068; Balick \& Heckman 1985 and NGC 7469; Wilson et al.1991). Part of the far-IR (FIR) luminosity of Seyferts is due to thermal reradiation of dust heated by hot OB stars (Rodriguez-Espinosa et al. 1987) and indeed, starbursts and Seyfert 2s have similar IRAS flux ratios and $\mathrm{H} \alpha$ luminosities (Dahari \& DeRobertis 1988). Seyfert 2s in general have stronger mid-IR (MIR; Edelson et al. 1987; Maiolino et al. 1995), FIR (Dahari \& De Robertis 1988), CO (Heckman et al.1989) and radio emission (Ulvestad \& Wilson 1989) than Seyfert 1s, indicating stronger star forming activity in their host galaxies.

Another important question in AGN research is whether observationally different types of AGN are intrinsically different or the same phenomenon. Recently, major effort has been put into unified models to explain the differences in terms of collimated radiation and orientation effects (e.g. Barthel 1989; Antonucci 1993). Specifically for Seyfert galaxies, it has long been been proposed that there is basically only one type of Seyfert nucleus with the optical spectroscopic division between types 1 and 2 dependent both on obscuration in and around the broad line region (BLR) and the luminosity of the central energy source (e.g. Lawrence \& Elvis 1982). As we will discuss in Sect. 6.2, there is now compelling evidence that in Seyfert 1s we see the compact nucleus and BLR directly, whereas in Seyfert 2s optically thick material, in the form of a thick disk, torus or an outflowing wind, blocks our direct view, and the ENLR along the minor axis of the torus is lit up by the soft X-ray/UV radiation from the nucleus in two oppositely directed cones. 
Although the nuclear torus is likely to be too small to be resolved, its outer parts e.g. in the form of a flattened dust distribution may be detectable. Because this material is expected to be redder than the surrounding stellar population, the geometry of the obscuring region should be apparent in colour maps. Evidence for this, in the form of a red distribution of continuum light perpendicular to the bipolar ENLR has been detected in NGC 5252 (Tadhunter \& Tsvetanov 1989; Kotilainen \& Prieto 1995) and NGC 5728 (Tsvetanov et al. 1996).

In Kotilainen \& Ward (1994), we discussed the general properties of the host galaxies of a sample of hard X-ray selected AGN, which are mainly Seyfert 1 nuclei. We found that the host galaxy colours are usually redder than in normal spiral bulges. The most likely explanation is a combination of dust obscuration and/or reradiation from hot dust. We detected colour gradients with the nucleus being redder than the bulge. There is a good correlation between the AGN and host galaxy luminosity. Most of the emphasis in studies of the nuclear regions of Seyferts has concentrated on the emission line properties (since integral field spectroscopy and emission line imaging provide the best tools to study the ENLR). Except for the recent work of Pogge \& De Robertis (1993), the continuum emission has been relatively neglected. In this paper we concentrate on the circumnuclear regions in Seyferts, rather than the integrated properties of the host galaxy. We present high resolution colour images of nine Seyfert galaxies (NGC 1068, NGC 3227, NGC 4151, NGC 7469, Mrk 3, Mrk 78, Mrk 348, Mrk 573 and NGC 1667). These images resolve the circumnuclear regions and reveal the existence of knots, elongations and rings not discernible in single filter images. We describe the observations in Sect. 2. In Sects. 3 and 4 we outline the data reduction procedures and methods used in the construction of the colour maps. In Sect. 5 we present the results for each galaxy, including comparison with available multiwavelength data. In Sect. 6 we discuss the relationship between Seyfert and starburst activity and the relevance of our results in the context of current unified AGN models. Conclusions are presented in Sect. 7. Throughout this paper we use Hubble constant of $H_{0}=50 \mathrm{~km} \mathrm{~s}^{-1} M p c^{-1}$, and a deceleration parameter $q_{0}=0$.

\section{Observations}

CCD images through broad band $B, V, R$ and $I$ filters were obtained of the nine galaxies, using a thick-coated, frontside illuminated blue-sensitive Tektronix $(520 \times 520)$ CCD camera located at the Cassegrain focus of the 2.5 $m$ Nordic Optical Telescope (NOT) on La Palma, during the nights of 12 and 14 November 1990 (NGC 1068, NGC 3227, NGC 4151 and NGC 7469) and 25 and 26 December 1992 (Mrk 3, Mrk 78, Mrk 348, Mrk 573 and NGC 1667). The pixel scale employed in each case was $0.20^{\prime \prime} \mathrm{px}^{-1}$. Conditions were photometric on 14 Novem- ber 1990 and 25 December 1992, with generally subarcsec seeing. On 12 November 1990 and 26 December 1992, conditions were poorer with seeing $1.0-2.3^{\prime \prime}$.

Table 1 gives details of the observations for each galaxy; it is formatted as follows: Column (1): the name of the galaxy; (2): the date of the observation; (3): the filter used, (4): integration time and (5): seeing during the observation. Field stars were present in the frames of NGC 7469, Mrk 3, Mrk 348, Mrk 573 and NGC 1667. For the other galaxies, seeing estimates were based on images of standard stars taken close in time before or after the galaxy observation.

Table 1. Journal of the observations

\begin{tabular}{|c|c|c|c|c|}
\hline Galaxy & Date & Filter & $\begin{array}{r}T_{\text {int }} \\
(\mathrm{sec})\end{array}$ & $\begin{array}{c}\text { FWHM } \\
\left({ }^{\prime \prime}\right)\end{array}$ \\
\hline (1) & (2) & (3) & (4) & (5) \\
\hline \multirow[t]{4}{*}{ NGC 1068} & 1990 Nov 14 & $B$ & 900 & 0.9 \\
\hline & & $V$ & 540 & 0.9 \\
\hline & & $R$ & 450 & 0.8 \\
\hline & & $I$ & 540 & 0.7 \\
\hline \multirow[t]{4}{*}{ NGC 3227} & 1990 Nov 14 & $B$ & 900 & 0.8 \\
\hline & & $V$ & 540 & 0.7 \\
\hline & & $R$ & 750 & 0.8 \\
\hline & & $I$ & 540 & 0.7 \\
\hline \multirow[t]{4}{*}{ NGC 4151} & 1990 Nov 14 & $B$ & 360 & 0.8 \\
\hline & & $V$ & 135 & 0.8 \\
\hline & & $R$ & 60 & 0.9 \\
\hline & & $I$ & 135 & 0.8 \\
\hline \multirow[t]{2}{*}{ NGC 7469} & 1990 Nov 12 & $V$ & 1200 & 2.3 \\
\hline & & $I$ & 900 & 1.5 \\
\hline \multirow[t]{4}{*}{ Mrk 3} & 1992 Dec 25 & $B$ & 1200 & 0.9 \\
\hline & & $V$ & 1200 & 0.9 \\
\hline & & $R$ & 900 & 0.8 \\
\hline & & $I$ & 900 & 0.8 \\
\hline \multirow[t]{3}{*}{ Mrk 78} & 1992 Dec 26 & $B$ & 1200 & 1.3 \\
\hline & & $V$ & 900 & 1.2 \\
\hline & & $I$ & 900 & 1.0 \\
\hline \multirow[t]{4}{*}{ Mrk 348} & 1992 Dec 25 & $B$ & 1800 & 0.9 \\
\hline & & $V$ & 1200 & 0.9 \\
\hline & & $R$ & 1200 & 0.8 \\
\hline & & $I$ & 1200 & 0.8 \\
\hline \multirow[t]{4}{*}{ Mrk 573} & 1992 Dec 26 & $B$ & 1200 & 1.3 \\
\hline & & $\bar{V}$ & 1200 & 1.2 \\
\hline & & $R$ & 900 & 1.1 \\
\hline & & $I$ & 900 & 1.0 \\
\hline \multirow[t]{4}{*}{ NGC 1667} & 1992 Dec 26 & $B$ & 900 & 1.3 \\
\hline & & $V$ & 900 & 1.2 \\
\hline & & $R$ & 900 & 1.1 \\
\hline & & $I$ & 900 & 1.0 \\
\hline
\end{tabular}

\section{Data reduction}

The raw CCD images were reduced using the KAPPA package at the STARLINK node in Cambridge (1990 data), and the ESO MIDAS software package at ESO, Garching (1992 data), with standard procedures, i.e. subtraction of the bias current level using a median averaged bias frame, dark current subtraction using dark frames of the same exposure time as the object frame, and flatfielding using a normalized median average of dome flatfield frames. Subtraction of the residual sky was performed by averaging the count levels in several areas away from the 
galaxy image. Cosmic rays and bad pixels were removed interactively. Multiple exposures were coadded after accurate alignment, to produce the final image. The photometry was calibrated against faint standard stars, observed just before or after the galaxies. An airmass correction was applied to remove the atmospheric extinction, but the frames were not corrected for the small amounts of galactic extinction. The calibrated photometry for NGC 3227, NGC 4151 and NGC 7469 is presented in Kotilainen et al. (1993). For the other galaxies, Table 2 lists the photometry in the $B, V, R$ and $I$ wavebands. We estimate a photometric accuracy of $0.03-0.05$ mag based on the standard star dispersion.

Note that at the time of the observations, the CCD camera used suffered from a substantial (up to 10\%) nonlinearity problem caused by a flaw in the amplifiers after the readout of the chip and before A/D conversion. This non-linearity has no dependence on colour or pixel position or time variation and was corrected for by a polynomial algorithm developed by Kjeldsen (1990). The corrected images are linear to better than $1 \%$ and any remaining non-linearity does not affect our conclusions.

Table 2. Photometry

$\begin{array}{lrcccr}\text { Galaxy } & \begin{array}{r}\text { Ap. } \\ \left({ }^{\prime \prime}\right)\end{array} & B & V & R & I \\ (1) & (2) & (3) & (4) & (5) & (6) \\ \text { NGC 1068 } & 3 & 13.54 & 12.76 & 11.98 & 11.64 \\ & 6 & 12.83 & 12.01 & 11.22 & 10.77 \\ & 9 & 12.44 & 11.60 & 10.83 & 10.27 \\ \text { Mrk 3 } & 12 & 12.17 & 11.32 & 10.56 & 9.94 \\ & 3 & 16.09 & 14.80 & 13.68 & 13.49 \\ & 6 & 15.41 & 14.19 & 13.10 & 12.73 \\ & 9 & 15.09 & 13.90 & 12.82 & 12.38 \\ \text { Mrk 78 } & 12 & 14.89 & 13.72 & 12.65 & 12.15 \\ & 3 & 16.77 & 16.22 & - & 14.67 \\ & 6 & 15.80 & 15.34 & - & 13.89 \\ \text { Mrk 348 } & 9 & 15.47 & 15.02 & - & 13.61 \\ & 12 & 15.30 & 14.84 & & 13.45 \\ & 3 & 17.14 & 16.03 & 15.13 & 14.64 \\ & 6 & 16.43 & 15.38 & 14.48 & 13.92 \\ \text { Mrk 573 } & 9 & 16.06 & 15.03 & 14.14 & 13.55 \\ & 12 & 15.79 & 14.80 & 13.91 & 13.30 \\ & 3 & 15.78 & 15.51 & 14.66 & 14.17 \\ & 6 & 15.01 & 14.74 & 13.90 & 13.43 \\ \text { NGC 1667 } & 9 & 14.68 & 14.42 & 13.59 & 13.10 \\ & 12 & 14.48 & 14.21 & 13.40 & 12.90 \\ & 3 & 16.25 & 15.94 & 14.99 & 14.32 \\ & 6 & 15.24 & 14.89 & 14.06 & 13.35 \\ & 9 & 14.71 & 14.33 & 13.56 & 12.85 \\ & 12 & 14.36 & 13.94 & 13.23 & 12.52\end{array}$

\section{Construction of the colour maps}

To produce the colour maps, the broad band images in different filters were first aligned to within a small fraction of a pixel. For the images of NGC 7469, Mrk 3, Mrk 348, Mrk 573 and NGC 1667, this was achieved using field stars as reference points. For the images of the other galaxies, there were no stars within the field. In these cases, the alignment was achieved using the galactic nucleus as the reference point. In both cases, we used the central pixel with the highest intensity as the reference point. The frames were also corrected for differences in the magnification factor between the different filters. Although determining the centroid of the light distribution is preferable to using just the central pixel as the reference point, any resulting misalignment is likely to be only a small fraction of a pixel, and so should not influence the results for structures covering a large number of pixels.

The flux calibrated images were then smoothed to the same (lowest) resolution, by application of a Gaussian filter with a width corresponding to the seeing profile appropriate for the lowest resolution frame. Then the frame corresponding to the longer wavelength was divided by the shorter wavelength frame. The resulting maps are plotted in logarithmic intervals, with darker shades indicating blue and lighter shades red emission. At the edge of the colour frames the noise dominates, but closer to the nucleus the colours are reliable.

To check that the structures apparent in the colour maps are not artefacts caused by, for example, different seeing conditions in the frames or errors in the alignment, we have constructed colour maps of the quasar 3C 273, which was observed during the November 1990 run. Reassuringly, neither 3C 273 nor the stars within its field show rings or other artefacts. Instead, for 3C 273 we find that the colour becomes gradually and smoothly bluer towards the nucleus. Also, the field stars in the other galaxy frames do not show rings or other features, but cancel out well in the colour image.

A problem in using broad band filters for continuum studies is the contamination from emission lines within the pass band. The $B$ filter is devoid of strong emission lines except for contribution from $\mathrm{H} \beta \lambda 4861$. The $V$ filter includes [OIII] $\lambda 4959,5007$ and some of the broad and narrow $\mathrm{H} \beta \lambda 4861$. The $\mathrm{R}$ filter includes a number of strong lines, $\mathrm{H} \alpha \lambda 6563$, [OI] $\lambda 6300,6364$, [NII] $\lambda 6548,6854$ and [SII] $\lambda 6717,6731$ lines. The $I$ filter is virtually free of emission lines, except for [O I] $\lambda 8446$ and [SIII] $\lambda 9069$ lines. Therefore, the $V$ and $R$ bands are most severely affected by emission lines, but the $B$ and $I$ filters are dominated by the continuum emission. This conclusion is supported by a literature search of EW's of major optical emission lines in the nuclear spectra of the sample galaxies. The average contribution of emission lines to the broad band filters are $5 \%(B), 17 \%(V$ and $R$ ), and $1 \%$ ( $I$ filter).

\section{Results}

There is a clear difference between the direct images obtained through the various filters. The $I$ band images display a smooth distribution of mainly evolved red stars, whereas the $B$ and $V$ images reveal the presence of 
absorbing dust, $\mathrm{H}$ II regions and young stars. In the $B-I$ maps of most galaxies, we detect clumpy, ring-like or elongated blue or red structures. Next we present as b/w figures (Plates 1-22) for each galaxy a blue ( $B$ band, except $V$ band for NGC 7469) direct image for morphological information and various colour maps.

\section{1. $N G C 1068$}

The Sb type Seyfert 2 galaxy NGC 1068 is the nearest object displaying both AGN and starburst activity, with $1^{\prime \prime}$ corresponding to a projected size of 110 pc. In Plate 1, we show its B band image. The nucleus exhibits a Seyfert 2 optical spectrum in direct light, but spectropolarimetry has revealed a broad, polarized component to the narrow emission lines (e.g. $\mathrm{H} \beta$ ), indicating the presence of an obscured Seyfert 1 nucleus (Antonucci \& Miller 1985; Miller et al. 1991). UV, optical and near-IR (NIR) observations of the bright inner $\left(\leqq 15^{\prime \prime}\right)$ disk indicate a young stellar spectrum with strong Balmer absorption and emission lines, suggesting a large population of B type stars and their associated H II regions, typical of a starburst (e.g. Bruhweiler et al. 1991). NGC 1068 is extraordinarily rich in molecular gas, with an estimated total $\mathrm{H}_{2}$ mass of $1.510^{10} M_{\odot}$ (Planesas et al. 1991). The MIR and FIR emission is extended (Braatz et al.1993; Cameron et al.1993) indicating that the IR emission originates in dust not solely heated by the AGN. The complex linear radio source of $\sim 7^{\prime \prime}$ in extent has two compact jets on opposite sides of the nucleus (Wilson \& Ulvestad 1983, 1987). The NLR is cospatial with the radio emission and displays an outflow in the direction of the jets, driven by interaction with radio plasma (Baldwin et al. 1987), characteristic of AGN activity. Both the NLR (Pogge 1989) and the ENLR (Unger et al.1992) share a conical shape aligned with the radio axis, indicative of photoionization by a nuclear radiation field collimated by the torus. A ROSAT X-ray image of NGC 1068 (Wilson et al.1992) reveals, in addition to a compact central source, larger scale emission, which is coincident with the outer edges of the optical disk and is probably due to SNRs, $\mathrm{X}$-ray binaries or a starburst driven wind, and not to electron scattering of the hidden Seyfert 1 nucleus.

The primary aim of this work is to use the colour maps to investigate the properties of the nucleus, and circumnuclear regions on kiloparsec scales. However, most of the host galaxy is also imaged, so we shall begin by briefly commenting on the large scale structures.

\subsubsection{Large scale structures in the $B-I$ map}

There are two previous studies of the large scale colour properties of NGC 1068. Schild et al. (1985) obtained CCD images in $B, V$ and $R$. Ichikawa et al. (1987) published ratios of photographic images, based on plates taken using $B, V$, and $i$ filters. Our large scale $B-I\left(70 \times 70^{\prime \prime}\right.$; $7.7 \times 7.7 \mathrm{kpc}$ ) map is shown in Plate 2. Our map agrees well with the $B-R$ image of Schild et al., excluding regions within $5^{\prime \prime}$ of the nucleus. The oval distribution of $\mathrm{H}$ II regions, centred on the nucleus and with major axis about $30^{\prime \prime}$, is clearly seen in Plate 2 . We see much more detail in the structure of the H II regions than Schild et al. because of our deeper imaging and better seeing.

There is an arc of blue resolved features NE-SW through the nucleus roughly parallel to the radio jet axis. These knots (labelled by Schild et al. 1985) are known to be related to sites containing luminous young stars (e.g. Bruhweiler et al. 1991; Truong \& Bruhweiler 1991). All the knots have similar spectra, so they should have comparable star formation ages. Our images cover the knots 2 to 4 (see Schild et al.). The colours of these knots within a $3^{\prime \prime}$ aperture are listed in Table 3. As for all the galaxies in Table 3, the colours include contribution both from the knots and the underlying old redder population. Therefore, the real colours of the knots must be even bluer than those reported here. The colours are in good agreement with Schild et al. (who used a $7^{\prime \prime}$ aperture), and confirm that they are sites of recent star formation. Knot 4 is extremely blue and could be a very recent star formation site, as supported by its Wolf-Rayet type optical spectrum (Bruhweiler et al. 1991).

Table 3. Colours of the maxima

$\begin{array}{lllccc}\text { Galaxy } & \text { Ap. }\left(^{\prime \prime}\right) & \text { Maximum } & B-V & V-R & V-I \\ (1) & (2) & (3) & (4) & (5) & (6) \\ \text { NGC 1068 } & 3 & \text { Knot } 2 & 0.39 & 0.75 & 0.88 \\ & 3 & \text { Knot } 3 & 0.54 & 0.76 & 1.23 \\ & 3 & \text { Knot } 4 & 0.24 & 0.57 & 0.75 \\ \text { NGC 3227 } & 2 & \text { Elongation } & 0.69 & 0.72 & 1.05 \\ & 1 & \text { SE } & 0.67 & 0.43 & 1.36 \\ \text { NGC } 4151 & 2 & \text { NW } & 0.55 & 0.60 & 1.28 \\ & 2 & \text { NE } & 1.01 & 0.47 & 0.99 \\ \text { NGC } 7469 & 2 & \text { SW } & 0.93 & 0.40 & 1.10 \\ & 2 & \text { S } & - & - & 1.06 \\ & 2 & \text { NE } & - & - & 1.13 \\ \text { Mrk 3 } & 2 & \text { NW } & - & - & 1.15 \\ & 2 & \text { W } & 0.78 & 1.03 & 1.40 \\ \text { Mrk } 78 & 2 & \text { nucleus } & 1.01 & 0.68 & 1.29 \\ & 3-9 & \text { ring } & 0.60 & - & 1.61 \\ \text { Mrk 348 } & 3 & \text { nucleus } & 1.11 & 0.90 & 1.39 \\ \text { Mrk 573 } & 3 & \text { NW } & 0.23 & 0.72 & 1.50 \\ & 3 & \text { SE } & 0.60 & 0.43 & 1.02 \\ \text { NGC 1667 } & 2 & \text { NE } & 0.46 & 0.91 & 1.60 \\ & 2 & \text { SW } & 0.29 & 0.93 & 1.57\end{array}$

The excellent correspondence of the colour properties of large scale structures, gives us confidence concerning the procedures used to produce our maps. We now turn to interpretation of the nuclear features. 


\subsubsection{Small scale structures in the $B-I$ map}

Unlike the previous work mentioned above, our data can be used to study details on scales of a few arcsecs. For this purpose we show the $B-I$ map of the innermost $20 \times 20^{\prime \prime}(2.2 \times 2.2 \mathrm{kpc})$ in Plate 3 . In the nuclear region on scales less than $10^{\prime \prime}$, there is an elongated blue structure, extending along PA $40^{\circ}$ for about $6^{\prime \prime}(660 \mathrm{pc})$. It peaks $\sim 1^{\prime \prime} \mathrm{NE}$ of the nucleus and has similar colours to the average of the $\mathrm{H}$ II region knots (Table 3), except $B-$ $V$, which is redder. These colours are much bluer than a typical $B-I$ index for a spiral bulge of $T=2(\sim 2.4)$, but redder than the average $B-I$ index for Seyfert 1 galaxies $(\sim 1.2)$. It is likely that the blue elongation is caused by scattered light from the nucleus of NGC 1068, which is superimposed on the stellar population of the nuclear region, which is redder. Therefore the observed colour index is an upper limit to the blueness of the nuclear source whose radiation is scattered.

Pogge \& De Robertis (1993) presented narrow band emission line and continuum imaging observations of NGC 1068. In the innermost region of their "UV/Red" (3600/6100 ̊) colour map, they find a number of blue features. The bluest region is offset from the nucleus by $\sim 0.8^{\prime \prime}$ in $\mathrm{PA} \sim 33^{\circ}$. The blue elongation is along the radio axis and roughly coincident with the peak of the [OIII] cone structure (Evans et al.1991), radio continuum NE peak (Wilson \& Ulvestad 1983) and the $10 \mu \mathrm{m}$ emission peak (Tresch-Fienberg et al.1987). Additionally, there is an extended, diffuse UV excess continuum component with a conical morphology. More complex situation is revealed by the recent HST UV and optical imaging of NGC 1068 (Macchetto et al.1994) who confirm the presence of the conical blue structure in their $\mathrm{UV} / \mathrm{U}$ continuum ratio map extending over $4^{\prime \prime}$ with quite uniform colour. The UV peak is displaced by $0.05^{\prime \prime}$ to north of the [OIII] peak and the UV continuum region envelopes the [OIII] emitting region. Intriguingly, they found a "twin-crescent" object SW of the cone. This object is highly polarized $(\sim 45 \%)$ and lies at the center of the overall polarisation pattern of the circumnuclear region and at the apex of the cones (Capetti et al.1995a,c). This structure probably straddles the real nucleus, but is not the torus, because it is misoriented with respect to the cones.

The morphology in Plate 3 is in good agreement with the structure observed by Pogge \& De Robertis (1993) and Macchetto et al. (1994) and with the structure in the imaging polarimetry of NGC 1068 by Miller et al.(1991) and Capetti et al. (1995a,c). We shall discuss the origin of this elongation in more detail, in conjunction with other galaxies, in the context of scattering of the nuclear light, in Sect. 6.2.

\section{2. $N G C 3227$}

The SAB pec galaxy NGC 3227 (a $B$ band image is shown in Plate 4) is interacting with a spheroidal companion NGC 3226. NGC 3227 has Seyfert type 1 spectrum (Salamanca et al.1994). but NGC 3226 shows no signs of activity, either AGN or star formation. The system is probably in the postcollision phase (Taniguchi et al. 1990). The X-ray luminosity of NGC 3227 is low for a Seyfert 1 galaxy (Ward et al.1987). It has a substantial amount of molecular hydrogen emission, based on NIR spectroscopy (Fischer et al. 1987) as well as strong millimeter CO emission (Blitz et al. 1986; Meixner et al. 1990). The resolved $\mathrm{CO}$ distribution has two intensity peaks straddling the nucleus, aligned roughly E-W and separated by $2^{\prime \prime}$ (Meixner et al.). A more extended component (with a diameter $17^{\prime \prime}$ ) lies SE-NW along a $30^{\circ}$ angle to the galactic major axis, the NW part is the site of most of the emission. In the maps of NGC 3227, $1^{\prime \prime}$ corresponds to $100 \mathrm{pc}$.

Our full scale $\left(70 \times 70^{\prime \prime} ; 7 \times 7 \mathrm{kpc}\right) B-I$ map (Plate 5$)$ shows a wealth of structure. Especially, there is a band of light (blue) colour, roughly in a shape of $\mathrm{Z}$ delineating the nucleus, indicating the geometry of the dust absorption in the galaxy. This geometry agrees well with that seen in the H I $21 \mathrm{~cm}$ absorption map by Mundell et al. (1995).

In Plate 6 , we show the $B-I$ image of the innermost $20 \times 20^{\prime \prime}(2 \times 2 \mathrm{kpc})$ of NGC 3227 . The colour map shows two blue maxima on either side of the nucleus (NW and $\mathrm{SE})$ with a separation $\sim 2^{\prime \prime}$.

Star forming regions are known to be associated with dense molecular clouds and dust. CO mapping indicates the presence of molecular clouds and hence the material available for star formation (Heckman et al.1989). Therefore, a correlation between the $\mathrm{CO}$ distribution and the colour maps would be expected if the blue maxima are caused by recent star formation. Indeed, we find a good correspondence between the CO map (Meixner et al. 1990) and our $B-I$ map (Plate 6 ), where two blue maxima are coincident with the $\mathrm{CO}$ emission peaks. The agreement is especially good for the SE maximum.

However, there is no direct evidence of ongoing star formation in the nuclear region of NGC 3227. On the other hand, the nucleus of NGC 3227 is strongly polarised, with a degree of polarisation rising smoothly from $\sim 1 \%$ in the red to nearly $3 \%$ in the blue (Schmidt \& Miller 1985), and essentially constant PA, both for the continuum and the broad lines. This polarisation can be best explained by interstellar dust scattering, where the dust is intermixed with the narrow line region (NLR) gas (Schmidt \& Miller). The PA of the polarisation is, interestingly, roughly parallel to the nuclear radio source axis (Ulvestad et al. 1981) and the optical major axis, suggesting that the dust clouds are distributed in the same plane as the overall galactic structure. Indeed, the double maxima are orientated along the major axis, and it is plausible that they represent scattering of the nuclear light in unobstructed directions. 
The colours of the blobs in a $1^{\prime \prime}$ aperture are given in Table 3. They are quite red, and not typical of young hot stars or scattered light, but the real colours must be bluer, since they include also the underlying structure, which is redder than the actual blue maximum. Note that the aperture used is very small (to avoid contamination from the other maximum and the nucleus) and the colours therefore prone to photon statistics errors.

\section{3. $N G C 4151$}

NGC 4151 is an SABab galaxy (de Vaucouleurs et al. 1991) and it is the best studied bright Seyfert 1 galaxy. A B band image of NGC 4151 is shown in Plate 7. Its redshift is 0.0033 and $1^{\prime \prime}$ corresponds to $100 \mathrm{pc}$ on the sky. The BLR is point-like but the NLR extends in a biconical structure $\sim 4^{\prime \prime}$ at $\mathrm{PA} \sim 60^{\circ}$ (Evans et al. 1993) and the ENLR is resolved into a jet-like feature with a series of high-ionization blobs covering $\sim 15^{\prime \prime} \mathrm{SW}$ and a fainter plume $5^{\prime \prime} \mathrm{NE}$ at $\mathrm{PA} \sim 50^{\circ}$ (e.g. Heckman \& Balick 1983; Perez et al. 1989). Extended X-ray emission at the brightest parts of the ENLR was discovered by Elvis et al. (1983). The radio structure is a collection of resolved sources (Harrison et al. 1986). The nuclear jets are at arcsec scale at $\mathrm{PA} \sim 70-80^{\circ}$ and misaligned with the ENLR by $\sim 30^{\circ}$, whereas at smaller scales, the radio component is elongated at $\mathrm{PA} \sim 57^{\circ}$, similar to the NLR and ENLR (Heckman \& Balick 1983; Pedlar et al. 1993).

In Plate 8 , we plot the $B-I$ map of the innermost $10 \times 10^{\prime \prime}(1 \times 1 \mathrm{kpc})$ of NGC 4151 . There is a strong colour gradient with the circumnuclear region being bluer than the rest of the bulge. This elongated blue structure is at $\mathrm{PA} \sim 60^{\circ}$ extending over $7^{\prime \prime}$ in diameter. The elongation is parallel to the small scale radio components and the NLR (Evans et al.1993). In the nuclear region we find two unresolved blue components symmetrically located each side of the nucleus (at $\mathrm{PA} \sim 80^{\circ}$ ) separated by $\sim 2^{\prime \prime}$. They are misaligned by $\sim 20^{\circ}$ with respect to the ionisation cones (Evans et al.1993) and the small scale radio axis. This structure is in good correspondence with the knots in the similar resolution radio map by Johnston et al. (1982).

The colours of the two maxima are given in Table 3 . The $B-V$ colours are surprisingly red; the other colours are consistent with normal spiral bulges. Since the real colours (without the underlying structure) must be bluer, these colours could be caused by a young stellar population or by scattered nuclear light. Again, with no firm evidence of ongoing star formation in the nucleus of NGC 4151, we prefer the latter possibility, but discuss the problems associated with this interpretation in Sect. 6.2.

\section{4. $N G C 7469$}

NGC 7469 (a $V$ band image is shown in Plate 9) is a well studied Seyfert 1 galaxy of Hubble type SAB(rs)a and redshift 0.0167 ( $1^{\prime \prime}$ corresponds to $490 \mathrm{pc}$ on the sky) ex- hibiting a circumnuclear starburst ring. It shows strong $3.3 \mu \mathrm{m}$ emission feature from dust heated to $T \geqq 300 \mathrm{~K}$, in a region between 1 and $3^{\prime \prime}$ from the nucleus, with the heating of the dust grains probably due to radiation from hot young stars in the circumnuclear H II regions (Cutri et al. 1984). NGC 7469 also exhibits the PAH emission features in the MIR spectrum (Roche et al.1991), common for starbursts. The $12.5 \mu \mathrm{m}$ emission is elongated $3^{\prime \prime}$ along $\mathrm{PA}=78^{\circ}$ probably representing unresolved integrated emission from the nucleus and two brightest starburst ring features (Keto et al. 1992). NGC 7469 has strong emission in the ${ }^{12} \mathrm{CO} J=1-0$ (Meixner et al.1990) and $\mathrm{H}_{2} \nu$ $=1-0 \mathrm{~S}(1)$ (Genzel et al.1995) lines within a few arcsec from the nucleus, providing evidence for a concentration of molecular gas.

A high resolution CO map of NGC 7469 (Meixner et al. 1990) shows, in addition to a CO peak coincident with the nucleus, a SW component $\sim 3.5^{\prime \prime}$ from the nucleus and an extension to the NW. In Plate 10, we present our $V-I$ map of the innermost $10 \times 10^{\prime \prime}(4.9 \times 4.9 \mathrm{kpc})$. The $V-I$ map shows an irregular ring of blue emission around the nucleus with a diameter $\sim 3^{\prime \prime}$. There are three notable blue maxima, in the $\mathrm{S}$ and in the NE and NW quadrants. The overall dimensions agree quite well with the $\mathrm{CO}$ emission (Meixner et al. ) although there is not a one-to-one correspondence. The SW CO plume is not reproduced, neither the NE maximum in the colour map.

A high resolution $\left(0.4^{\prime \prime} \mathrm{FWHM}\right)$ radio map at $6 \mathrm{~cm}$ of NGC 7469 (Wilson et al.1991) is dominated by a strong compact source centered on the Seyfert nucleus. Much of the remaining radio halo $\left(\sim 8^{\prime \prime}\right.$ diameter $)$ is concentrated into an annular structure of diameter $\sim 3^{\prime \prime}$, with bright maxima to the $\mathrm{NE}$ and SW. An [OIII] $/ \mathrm{H} \alpha$ image of Wilson et al. shows that $\mathrm{H} \alpha$ is extended around a bright peak coincident with the continuum nucleus. The [OIII] emission is much more centrally concentrated with a plume to the $\mathrm{N}$.

Mauder et al.(1994) presented high resolution, speckle masking images at three optical wavelengths (5400$6700 \AA$ ) with the central source subtracted. The images show a ring shaped emission region with a radius of $1.3^{\prime \prime}$ and a lumpy structure with three main maxima at PA $\sim 40,245$ and $320^{\circ}$. The maxima in our $V-I$ image (Plate 10) correspond well with those in the radio, emission line and red continuum images. There is an especially good coincidence for the NE peak. The agreement for the SW peak is less good, but we have to remember the much higher resolution in the radio map. Also, the overall dimensions are in exact agreement. This similarity between the radio and optical indicates that the circumnuclear structure seen in the continuum colour map represents direct emission from hot stars in the ring, i.e. a starburst.

The colours of the blue circumnuclear blobs in a $2^{\prime \prime}$ aperture are given in Table 3 . The $V-I$ colours are remarkably uniform and close to normal old stellar 
population colours. Again, the real colours without the underlying structure must be bluer.

\subsection{Mrk 3}

Mrk 3 is an S0 type galaxy (Jenkins 1981; Wagner 1987) with a Seyfert 2 nucleus (Koski 1978) at a redshift of $0.0134 ; 1^{\prime \prime}$ corresponds to $410 \mathrm{pc}$ on the sky. A $B$ band image is shown in Plate 11. The X-ray spectrum of Mrk 3 (Awaki et al.1990) shows a heavy absorbing column $\left(N_{\mathrm{H}}=5.910^{23} \mathrm{~cm}^{-2}\right)$ and a strong Fe $\mathrm{K}$ line. This can be explained by absorption in and scattering off an obscuring torus. Awaki et al. suggest Mrk 3 being an intermediate case between less obscured (e.g. Mrk 348) and completely absorbed (e.g. NGC 1068) Seyfert 2s.

The H I mass is $\geq 710^{9} M_{\odot}$ (Pedlar et al. 1984), large for ellipticals. This may be due to interaction with UGC 03422, located $6^{\prime} \mathrm{NW}$ along $\mathrm{PA}=155^{\circ}$, although there is no evidence for this in direct optical images (Wagner 1987). At $5 \mathrm{GHz}$, Mrk 3 exhibits a pair of highly collimated jets perpendicular to the major axis of the galaxy, $2^{\prime \prime}$ in extent along $\mathrm{PA}=84^{\circ}$ (Kukula et al.1993). Embedded in the jets are compact components, the western end terminating in a bright lobe containing a hotspot. Mrk 3 has a dust-dominated steep spectrum in the NIR (Edelson \& Malkan 1986), high optical polarisation (1-2\% for lines and continuum at PA $=130^{\circ}$; Schmidt \& Miller 1985 ) and shows broad lines in polarised light (Miller \& Goodrich 1990).

Recent HST imaging by Capetti et al. (1995b) revealed a striking spiral shape of the ionized gas emission, with a large number of resolved knots in a fainter extended region. The brightest part of the spiral is confined into a cone of $\sim 40^{\circ}$ full opening angle, and its morphology is similar to the radio (Kukula et al.1993). The optical emission lines are blue asymmetric, indicating absorption by dust surrounding the NLR clouds. The nuclear emission thus seems to be heavily absorbed in the line of sight, while the ionizing flux is intrinsically not strongly collimated.

The $B-I$ image of the central $20 \times 20^{\prime \prime}(8.2 \times 8.2 \mathrm{kpc})$ region of Mrk 3 is shown in Plate 12. The central region is redder than the surrounding bulge at PA $\sim 100^{\circ}$ with extent $\sim 11^{\prime \prime}$. Within this red elongation, there is an arclike blue extension, with two main blue maxima, roughly elongated at $\mathrm{PA}=95^{\circ}$. The $\mathrm{W}$ maximum is bluer at $\sim 1.3^{\prime \prime}$ from the nucleus, while the $\mathrm{E}$ maximum is at $0.8^{\prime \prime}$ distance from the nucleus. In the $\mathrm{E}$, the elongation curves towards larger PA, fading away at $\sim 4.6^{\prime \prime}$ from nucleus. The double structure is roughly aligned with the radio axis $\left(85^{\circ}\right.$; Ulvestad \& Wilson 1984). The opening angle of the "cone" agrees well with that measured from the emission lines (Capetti et al.1995b), and the curvature of the blue continuum is similar ( $\mathrm{S}$-shaped) to that seen in the emission lines (Capetti et al. 1995b) and in the radio (Kukula et al. 1993). Most likely, the blue structure is scattered light from the nucleus following the biconi- cal structure in Mrk 3. Outside of the blue emission, the colour is typical of galactic bulges, displaying no sign of star forming regions in its host galaxy, rendering the possibility of jet-induced star formation unlikely.

Pogge \& De Robertis (1993) presented a "UV/Red" colour map of Mrk 3, which revealed two distinct structures: a pair of UV (blue) knots on either side of the nucleus along $\mathrm{PA}=85^{\circ}$, separated by $1.5^{\prime \prime}$, and a diffuse blue component with a distinct bi-conical morphology extending out to $5^{\prime \prime}$ on either side of the nucleus along PA $=114^{\circ}$. Our $B-I$ map is in good agreement with their map, with the PA's agreeing within the errors. We discuss the origin of these blue maxima in Sect. 6.2.

The $V-R$ map of Mrk 3 (Plate 13), which resembles an $[\mathrm{OIII}] / \mathrm{H} \alpha$ excitation map, shows a spiral structure in the outer regions with a radius $\sim 4^{\prime \prime}$ from the nucleus, in agreement with Capetti et al. (1995b) results. Interestingly, there is also a red double structure in the nuclear region, which corresponds well to the blue maxima in the $B-I$ map (Plate 12). The colours of the maxima in a $2^{\prime \prime}$ aperture are given in Table 3 . The $B V R I$ colours are redder than for the NGC 1068 elongation, but still in agreement with scattering, being contaminated by the underlying red stellar population.

\section{6. $M r k ~ 78$}

Mrk 78 is a Seyfert 2 galaxy at redshift $0.038\left(1^{\prime \prime}\right.$ equals $1.15 \mathrm{kpc}$ ). Plate 14 shows its $B$ band image. At $2 \mathrm{~cm}$, Mrk 78 shows a triple structure, with a bright core and fainter components to the $\mathrm{E}$ and $\mathrm{W}$, parallel to the NLR axis (Pedlar et al.1989). There is no detailed morphological correlation, however, between the radio and the ENLR (Unger et al.1987), with the axes misaligned by $\sim 25^{\circ}$ (Capetti et al. 1994). This misalignment can be caused by the ENLR being in a different plane from the rest of the galaxy, or by different covering factor $\mathrm{E}$ and $\mathrm{W}$ of the nucleus (Pedlar et al. 1989).

The [OIII] emission is biconical with opening angle $\sim 40^{\circ}$ extended along the radio axis at $\mathrm{PA}=67^{\circ}\left(3.5 \times 6.0^{\prime \prime}\right.$ in extent; Capetti et al.1994). There is also a resolved obscuring zone close to the center, elongated E-W, with $\sim 0.8^{\prime \prime}$ extent and displaced from the center of the cone by $\sim 0.2^{\prime \prime}$. The lack of far-UV emission, comparison with the FIR emission, and photon budget arguments indicate a strongly anisotropic nuclear source. Capetti et al. suggest that obscuring dust (both on pc and kpc scales) prevents UV radiation to escape along our line of sight, while the stellar continuum remains largely unobscured.

We present our $B-I$ map in Plate 15. It shows the nucleus as redder than the bulge of the galaxy. The reddest region close to the nucleus is slightly extended $\left(1.1 \times 1.7^{\prime \prime}=\right.$ $1.3 \times 2.0 \mathrm{kpc}$ ) along $\mathrm{PA} \sim 330^{\circ}$. This extension is almost perpendicular to the E-W [OIII] morphology (Capetti et al. 1994), and may show the location of the obscuring dust in this galaxy. There is also an arc-like blue region forming 
a broken ring from $\mathrm{E}$ through $\mathrm{N}$ to $\mathrm{SW}$ at a distance $\sim 3^{\prime \prime}$ from the nucleus. Again, although this could be a star forming region around the nucleus, or show a gradient in the stellar population, there is no direct evidence for this. A more appealing possibility is that it represents a scattering mirror which is less efficient or further away from the nucleus than in the cases of NGC 1068, Mrk 3 and Mrk 573 (see Sect. 6.2). The colours of the blue ring are redder in $V-I$, but bluer in $B-V$ than for the NGC 1068 elongation (Table 3).

\section{7. $M r k 348$}

Mrk 348 is an almost face-on S0/a-sb type galaxy (Huchra 1977) with a Seyfert 2 nuclear spectrum (Koski 1978). The $B$ band image is shown in Plate 16 . The velocity field of the neutral hydrogen and tidal plumes indicate an extensive tidal perturbation by a companion galaxy, NGC 266 (Simkin et al. 1987). Its small reddening $(E(B-V) \leq 0.10$ both for lines and continuum) is mainly produced in our Galaxy. At a redshift of Mrk 348 (0.014), 1" corresponds to $420 \mathrm{pc}$ projected distance on the sky.

Spectropolarimetry has revealed the presence of broad wings to the permitted lines in Mrk 348 (Miller \& Goodrich 1990). The radio structure is linear on a scale of 0.2" (Neff \& de Bruyn 1983; Ulvestad \& Wilson 1984). There is also an extended distribution of ionized gas in the nuclear region (Bergeron \& Durret 1987). The $2-10 \mathrm{keV}$ luminosity of Mrk 348 is a factor of 30 brighter (Warwick et al. 1989) and the $\mathrm{Fe} \mathrm{K} \alpha$ line an order of magnitude fainter (Koyama et al.1989) than in NGC 1068. Probably Mrk 348 is seen directly through much less column density than in the case of NGC 1068.

We present our $B-I$ map of the central $20 \times 20^{\prime \prime}$ $(8.4 \times 8.4 \mathrm{kpc})$ of Mrk 348 in Plate 17. It shows the nucleus as redder than the near-nuclear region. At larger distances, the host galaxy appears again as red. Between these, there is an intermediate blue ring-like region, $\sim 3^{\prime \prime}$ in diameter elongated at $\mathrm{PA} \sim 15^{\circ}$. Again, although the map of Mrk 348 is less clear than for the other galaxies, this blue structure may be related to scattering of the nuclear light, with less efficient "mirrors" than in the other galaxies studied.

\subsection{Mrk 573}

Mrk 573 is a Seyfert 2 galaxy at a redshift of 0.017 ( $1^{\prime \prime}$ corresponds to $510 \mathrm{pc}$ projected distance on the sky). Plate 18 shows its $B$ band morphology. The extinction towards the nucleus, $E(B-V) \sim 0.3$, is probably mostly intrinsic to the galaxy. It has a triple radio source at $6 \mathrm{~cm}$ with two lobes of similar luminosity at distance $\sim 1.5^{\prime \prime}$ from the nucleus along the radio axis at $\mathrm{PA}=125^{\circ}$ (Ulvestad \& Wilson 1984). Narrow band [OIII] and $\mathrm{H} \alpha$ images show an elongated emission line nebulosity extending about $10 \times 15^{\prime \prime}$ along the radio axis, and two oppositely directed cones of ionising radiation (Haniff et al. 1991; Tsvetanov \& Walsh 1992). The $\mathrm{H} \alpha$ peak coincides with the continuum peak, but the [OIII] maximum is shifted $4^{\prime \prime}$ along the radio axis to SE (Whittle et al. 1988; Tsvetanov \& Walsh 1992). Perpendicular to the line image elongation there is a lowionisation valley through nucleus at $\mathrm{PA} \sim 40^{\circ}$ (Tsvetanov \& Walsh 1992).

Kinney et al. (1991) compared the number of ionising photons seen by the ENLR gas to the number of ionising photons seen in Earth for a sample of Seyfert 2 galaxies. For Mrk 573, they found a ratio $\sim 1$. However, corrected for extinction and biconical covering factor, the ratio becomes $\geq 5$ (Tsvetanov \& Walsh 1992), providing strong evidence for anisotropy of the radiation field.

Pogge \& De Robertis (1993) presented a "UV/Red" map of Mrk 573, where they find a biconical structure oriented at $\mathrm{PA}=124^{\circ}$ with an opening angle $\sim 60^{\circ}$. They also find a curved feature $3.5^{\prime \prime} \mathrm{SE}$ of the nucleus and a similar feature $4.5^{\prime \prime} \mathrm{NW}$. Both are coincident with [OIII] emission line features, but outside the radio continuum lobes. Recently, Pogge \& De Robertis (1995) obtained narrow band images in five emission lines and a continuum band. In addition to the features mentioned above, which they found to be elongated perpendicular to the radio axis and rotated following the spiral arms rotation, they found two further arc-like structures at $1.7^{\prime \prime} \mathrm{SE}$ and $2.2^{\prime \prime} \mathrm{NW}$ of the nucleus at PA $124^{\circ}$, bracketing the radio lobes. Based on the morphology and ionisation gradient, Pogge \& De Robertis (1995) suggest that the inner pair of features is reminiscent of edge-brightened bow shocks where the emission line gas is interacting with the radio plasma from the AGN, while the outer pair may represent episodic ejection of plasma from the nucleus.

We present our $B-I$ map of the central $20 \times 20^{\prime \prime}$ $(10.2 \times 10.2 \mathrm{kpc})$ of Mrk 573 in Plate 19. It shows two blue maxima on either side of the nucleus with a separation of $\sim 7^{\prime \prime}$ at $\mathrm{PA} \sim 135^{\circ}$ and a red "bridge" perpendicular to them crossing the nucleus. The double structure is roughly parallel to the radio axis $\left(\mathrm{PA}=124^{\circ}\right.$; Ulvestad \& Wilson 1984) but the blue component is more extended than the radio continuum emission. There is also a tightly wound spiral arm structure tracing an elliptical ring with major axis $\sim 20^{\prime \prime}$ aligned at $\mathrm{PA} \sim 10^{\circ}$. Most likely, the blue maxima define the geometry of the radiation cones, with the red bridge indicating the large scale obscuring material across the nucleus. While the blue maxima could alternatively be understood as arising from star formation triggered by the radio jet, there is no sign of young blue stars in the continuum images. Furthermore, the ENLR line ratios indicate an AGN-type ionising spectrum. Note, however, that the $10 \mu \mathrm{m}$ flux of Mrk 573 is relatively larger than in typical Seyfert 2s, suggesting that a starburst component may contribute in the nucleus (Maiolino et al. 1995). There is a good general correspondence between the maps of Pogge \& De Robertis $(1993,1995)$ and our $B-I$ map (Plate 19); except that we can resolve the 
blue maxima, which are more diffuse and situated further away from the nucleus than in NGC 1068 and Mrk 3. For discussion of the blue maxima, see Sect. 6.2. The colours of the blue maxima in a $3^{\prime \prime}$ aperture are given in Table 3. They are bluer in $B-V$ and $V-R$ than those of the NGC 1068 elongation, and in good agreement with arising from scattering.

\section{9. $N G C 166^{r}$}

Except for its supernova 1986N, NGC 1667 has not been studied in detail so far. It is a relatively isolated type Sc galaxy with a Seyfert 2 nucleus at $z=0.0153 ; 1^{\prime \prime}$ corresponds to $470 \mathrm{pc}$ on the sky. A $B$ band image of NGC 1667 is shown in Plate 20. Evidence for star formation activity in its host galaxy comes from starburst-like FIR colours, slightly extended MIR emission, and optical morphology and emission line ratios (see references in Heckman et al.1989). The radio emission is diffuse and single-component (Ulvestad \& Wilson 1989), indicating that most of the radio emission in NGC 1667 does not arise from the AGN.

We present our large scale $B-I$ map $\left(60 \times 60^{\prime \prime}=\right.$ $28 \times 28 \mathrm{kpc})$ of NGC 1667 in Plate 21. The image delineates the spiral structure of this galaxy, the individual $\mathrm{H}$ II regions in the spiral arms, and the geometry of dusty material, as in the case of NGC 3227 (Plate 5). Note especially the red "bubble" extending up to $9^{\prime \prime} \mathrm{N}$ of the nucleus. Plate 22 shows the $B-I$ map of the central $20 \times 20^{\prime \prime}$ $(9.4 \times 9.4 \mathrm{kpc})$ nuclear region. In addition to the innermost spiral arm, there is a red double structure across the nucleus with a separation $\sim 3^{\prime \prime}$ at $\mathrm{PA} \sim 45^{\circ}$. The colours of these red maxima are given in Table 3. Although this red structure could be related to the material obscuring a Seyfert 1 nucleus, we defer further discussion, but encourage finding direct evidence of anisotropy in this little studied Seyfert 2.

\section{Discussion}

\subsection{Evidence for star formation activity}

A significant fraction of Seyfert galaxies show evidence for star formation in the circumnuclear regions (Wilson 1988). It appears that star formation is more important for Seyfert 2s than 1s (Mulchaey et al. 1994), although this may be a selection effect, since most Seyfert $2 \mathrm{~s}$ were discovered in Markarian surveys, biased toward blue colours, such as would arise from ongoing star formation.

The narrow-line ionized gas in Seyferts has several extranuclear components. Firstly, the NLR close to the nucleus has either a smooth morphology centered on the nucleus or, more often, a complex filamentary structure with a bipolar or linear appearance. This gas is probably photoionized by the continuum photons from the AGN, as evidenced by the emission line ratios, and it sometimes exhibits large noncircular kinematics. The second component consists of the ENLR (Unger et al.1987). The low velocity dispersion, orderly velocity field and high excitation indicate that the ENLR is ambient gas in the disc or halo of the galaxy photoionized by the (anisotropic) nuclear radiation field. The last component of ionized gas is knotty or clumpy and is usually associated with spiral arms or circumnuclear rings. The emission line ratios indicate photoionization by OB stars in giant $\mathrm{H}$ II regions and this gas is kinematically part of the rotating disk of the galaxy. In a minority of Seyferts, this component includes a circumnuclear starburst on scales from a few hundred pc to a few kpc. The colour maps presented in this paper relate mainly to this component.

Similarly, nonthermal radio sources in Seyferts can be divided into two morphologies. The majority have linear (double or triple) morphology straddling the optical nucleus and are probably fuelled by the AGN. The second, less frequent, class have diffuse or blob-like morphology. These are associated with the star forming regions seen in the optical and presumably represent the integrated emission from multiple SNRs or free-free emission (Miley et al. 1985; Edelson et al. 1987).

There is also diversity in the MIR and FIR spectra of Seyferts. Many Seyfert 2s exhibit prominent dust features in the $8-13 \mu \mathrm{m}$ spectra similar to starbursts, whereas Seyfert 1s generally lack them (Roche et al.1991), except those with circumnuclear starbursts, e.g. NGC 1365, NGC 7469 and NGC 7582. Many Seyfert 2s have FIR colours similar to starburst galaxies, whereas Seyfert 1s resemble quasars (Miley et al.1985). The MIR $(\sim 10 \mu \mathrm{m})$ emitting region in many Seyferts is spatially extended over kpc scales, necessitating a distributed heating source, probably young stars (Edelson et al. 1987).

The only galaxy in our sample showing clear circumnuclear starburst activity is NGC 7469. The diffuse radio morphology, IRAS colours and radio spectral index are typical of starburst galaxies (Wilson et al. 1986; Condon \& Broderick 1988). The emission line morphology is different from the aligned NLR often found in Seyferts (e.g. Haniff et al. 1988) and the emission line ratios all argue that much of the star formation occurs in the ring of diameter $3^{\prime \prime}$ around the Seyfert nucleus. This ring is clearly resolved in our $V-I$ colour map (Plate 10).

Can any of the other detected features in our sample be due to star formation? In NGC 1068, NGC 4151, Mrk 3 and Mrk 573, the radio structure is linear while the CO structure of NGC 1068 and NGC 4151 is diffuse and not peaked near the nucleus. For these galaxies, a more attractive explanation is scattering of the nuclear light along the radio axis (see Sect. 6.2). The radio structure of NGC 3227 is diffuse as in starbursts and the relatively good correspondence between the $\mathrm{CO}$ emission and the $B-I$ map and the colours of the blue maxima could be due to star formation. However, there is no direct evidence of star formation occurring in the circumnuclear region of 
NGC 3227, while other evidence (see Schmidt \& Miller 1985) strongly point towards scattering.

\subsection{The blue components and their implications for the unified models of Seyfert galaxies}

The colour images presented in this paper offer a new, independent method to test the unified models of AGN, and the technique should be applied to a larger sample. Unified models of AGN have become recently increasingly popular (e.g. Barthel 1989; Antonucci 1993). In these models, all Seyferts have the same basic structure but appear as either type 1 or 2 (or intermediate types) depending on obscuration and their orientation with respect to our lineof-sight.

Optical spectropolarimetry of NGC 1068 (Antonucci \& Miller 1985) showed that this archetypal Seyfert 2 galaxy has polarised broad emission lines, characteristic of a Seyfert 1 nucleus obscured from our direct view by an optically and geometrically thick torus (see also Krolik \& Begelman 1986). A fraction of the nuclear luminosity is scattered (polarised) into our line of sight by electrons in a tenuous, warm gas along the axis of the torus. The commonly accepted view now is that the distinction between broad and narrow lined AGN may simply be due to our viewing angle. In Seyfert 1s, we look along the axis of the obscuring torus, and directly see the nucleus and the BLR, whereas in Seyfert 2s the torus blocks our direct view of the nucleus, which can only be seen in reflected polarised light. Several other Seyfert 2 galaxies (e.g. Miller \& Goodrich 1990; Tran et al. 1992; Kay 1994) indeed have broad lines in polarised light. In addition, the presence of broad NIR lines in many Seyfert 2s reveals highly obscured BLRs (e.g. Blanco et al. 1990; Nakajima et al.1991; Goodrich et al. 1994; Ruiz et al. 1994).

In the unified model, the ionising nuclear radiation is collimated by the torus and escapes anisotropically along the torus axis, resulting in two oppositely directed cones. The ENLR in Seyferts is indeed preferentially aligned along the radio source (torus) axis (see Wilson \& Tsvetanov 1994). In several cases, [OIII] $/ \mathrm{H} \alpha$ images directly show the conical or bi-conical geometry of the high excitation gas (e.g. Pogge 1989; Tadhunter \& Tsvetanov 1989; Haniff et al.1991; Tsvetanov \& Walsh 1992) and the cone regions have spectra consistent with photoionization by the nuclear continuum.

Other lines of evidence also support this model. The ionizing photon flux required to produce the line emission inferred from the ionization state and energy balance of the gas in the NLR and ENLR is higher than that inferred from directly observed continuum in Seyfert 2s (e.g. Wilson et al. 1988; Kinney et al.1991), indicating that the NLR gas sees a stronger ionising source than observed from Earth. This collimated ionising radiation could result either from dust obscuration of an isotropic source or from an intrinsically anisotropic source (Acosta-Pulido et al.1990). Finally, hard X-ray emission and the $\mathrm{Fe} \mathrm{K} \alpha$ $\mathrm{X}$-ray line has been found in several Seyfert 2s (e.g. Koyama et al.1989; Warwick et al.1989; Awaki et al. 1990). The inferred absorption column densities are constant in time and in the range $N_{\mathrm{H}}=10^{22}-10^{24} \mathrm{~cm}^{-2}$ for all Seyfert 2s except NGC 1068, therefore, hard X-rays can pass through the torus (Krolik \& Lepp 1989). In NGC 1068, the column density is much larger, and the detected hard X-rays are due to a scattered nuclear component.

What is then the origin of the blue extensions and maxima visible in the colour maps, most clearly in the case of NGC 1068, NGC 3227, Mrk 3 and Mrk 573? They are unlikely to be intrinsically blue regions of star formation because there is no direct evidence for this in the continuum images (although see Macchetto et al.1994 for probable star clusters in the near nuclear region of NGC 1068), and because the morphology of the regions is diffuse, unlike the sharp boundaries seen e.g. in the $\mathrm{OB}$ associations further out in the disk of NGC 1068. Optical synchrotron emission from the radio jets is also unlikely, because there is no detailed correspondence between the blue maxima and the radio hot spot structure and the extended blue continuum is more extended than the radio continuum. The most likely remaining alternative is scattering of light from the Seyfert nucleus by dust or electrons. In either case the scattered continuum would appear blue, but more so for dust scattering because of the wavelength dependence. For NGC 1068, electron scattering in the nucleus is preferred, because of the wavelength-independent polarisation in the UV (Code et al.1993), but in the circumnuclear region dust also contributes (Miller et al.1991).

The blue knots in NGC 1068 and Mrk 3 are significantly offset from the nuclei, as are their [OIII] peaks. In Mrk 573, on the other hand, the blue maxima are even further away and lie beyond the inner radio continuum structure, whereas the [OIII] emission peaks on the nucleus. Similarly, NGC 1068 and Mrk 3 are classical examples of Seyfert 2 nuclei with polarised broad lines revealed by spectropolarimetry indicating a hidden Seyfert 1 nucleus (Antonucci \& Miller 1985; Miller \& Goodrich 1991), whereas Mrk 573 has only slightly polarized broad $\mathrm{H} \beta$ line (Kay 1994) and it does not show prominent blue knots very near the nucleus (although see the inner pair of blue arcs in Pogge \& De Robertis 1995). The off-center blue continuum knots may thus be "mirrors" scattering the nuclear light. A tentative size of the scattering region of $\sim 1.0-1.5 \mathrm{kpc}$ has been derived for NGC 1068 from multiaperture polarimetry (Antonucci et al. 1994). It may be that Mrk 573 lacks a strong scattering mirror, with most of the scattering distributed over a larger region, producing correspondingly weaker polarisation. In NGC 1068 and Mrk 3 there are unobstructed clouds close enough to the nucleus to produce strong polarisation. This is confirmed for NGC 1068, where there is a strongly polarised region NE of the nucleus (Miller et al.1991) with the 
orientation of the polarisation vector perpendicular to the line of sight to the nucleus, coinciding with the extended blue feature seen in our colour map. Similar imaging polarimetry and spectropolarimetry of Mrk 3 and Mrk 573 would be needed to resolve the question for them.

In the simplest scattering model, an optically thick molecular torus surrounds the BLR to obscure the ionizing photons, and the difference between objects with (Seyfert 1) or without (Seyfert 2) BLR depends solely on our line of sight to the torus. Is this model valid for the blue knots seen in Seyfert type 1 galaxies NGC 4151 and NGC 3227, in which we see the BLR directly? Emission line imaging of NGC 4151 by Evans et al. (1993) and Boksenberg et al. (1995) indicates that our line of sight lies outside the ionisation cones, and the central BLR should not be visible directly through the thick torus. Evans et al. and Boksenberg et al. considered several possibilities to reconcile the geometry of NGC 4151 with unified models, and prefer an optically thick torus surrounded by lower density atmosphere that collimates the ionising radiation but leaves the nucleus relatively unobscured. Another model was proposed by Robinson et al.(1994), who suggested that ionising radiation escapes from the nucleus in a broad cone (opening angle $\sim 120^{\circ}$ ), inclined close to the galactic disk. The ENLR is formed as the intersection of the radiation cone with the disk, and the wide opening angle accounts for the misalignment between the ENLR and the nuclear radio source. In their model, the opaque torus required to collimate the radiation field is composed of bar-driven inflow of dust and gas toward the nucleus.

Mrk 78 and Mrk 348 exhibit only weakly resolved blue structures outside nucleus. Do all Seyfert $2 \mathrm{~s}$ then have hidden Seyfert 1 nuclei? Miller \& Goodrich (1990) calculated the expected polarisations from randomly oriented scattering cones, and found that polarisations up to over $50 \%$ should be common. While such high polarisations have not been observed, this may be understood in the unified model, if scattering occurs close to the nucleus, and the scattering region itself is largely obscured. Especially in highly inclined galaxies, the dust lanes in the host galaxy can block the view to the scattering region. The remaining observed polarisation would also be strongly diluted by starlight. Similarly, our failure to convincingly detect the scattering region in these "good candidates for hidden Seyfert 1s" may mean that the electron scattering region is not present, not resolved and/or does not have sufficient optical depth to produce notable colour gradients.

\section{Conclusions}

Colour maps provide a new, powerful method to reveal star formation and scattering in the nuclear region of Seyfert galaxies. We have detected ring-like or elongated blue structures in the $B-I$ (or $V-I$ ) maps of several Seyfert galaxies and found a good agreement with previous spectroscopic and multifrequency studies of these galaxies. In NGC 1068, NGC 3227, NGC 4151, Mrk 3 and Mrk 573, we interpret the clear colour structure as arising from scattering of the nuclear light from electrons or dust. In Mrk 78 and Mrk 348 the structure is less clear, and may indicate that the scattering mirrors are less efficient and/or further away from the nucleus than in the other galaxies. In NGC 7469, there is a good agreement with the integrated molecular $\mathrm{CO}$ gas emission and radio continuum structure indicating circumnuclear star formation in a ring or a disk around the Seyfert nucleus.

Colour maps in multiple, emission line-free windows, in UV as well as in the optical, and detailed spectrophotometry would be valuable to confirm the presence of the detected elongations and to probe the physical conditions in more detail. In a forthcoming paper, we shall analyse broad band data of a further, larger sample of candidates of Seyfert 2s with obscured BLRs, obtained recently at the NOT.

Acknowledgements. We thank Malcolm Bremer for help with the observations of December 1992. This work was started when both authors were at the Institute of Astronomy, Cambridge. JKK thanks ESO for a postdoctoral fellowship, during which most of this work was done.

\section{References}

Acosta-Pulido J.A., et al., 1990, ApJ 365, 119

Antonucci R.R.J., 1993, ARA\&A 31, 473

Antonucci R.R.J., Miller J.S., 1985, ApJ 297, 621

Antonucci R., Hurt T., Miller J.S., 1994, ApJ 430, 210

Awaki H., Koyama K., Kunieda H., Tawara Y., 1990, Nat 346, 544

Baldwin J.A., Wilson A.S., Whittle M., 1987, ApJ 319, 84

Balick B., Heckman T., 1985, AJ 90, 197

Barthel P.D., 1989, ApJ 336, 606

Bergeron J., Durret F., 1987, A\&A 184, 93

Blanco P.R., Ward M.J., Wright G.S., 1990, MNRAS 242, 4P

Blitz L., Mathieu R.D., Bally J., 1986, ApJ 311, 142

Boksenberg A., Catchpole R.M., Macchetto F., et al., 1995, ApJ 440, 151

Braatz J.A., Wilson A.S., Gezari D.Y., Varosi F., Beichman C.A., 1993, ApJ 409, L5

Bruhweiler F.C., Truong K.Q., Altner B., 1991, ApJ 379, 596

Cameron M., Storey J.W.V., Rotaciuc V., et al., 1993, ApJ 419, 136

Capetti A., Macchetto F., Sparks W.B., Boksenberg A., 1994, ApJ 421,87

Capetti A., Axon D.J., Macchetto F., Sparks W.B., Boksenberg A., 1995a, ApJ 446, 155

Capetti A., Macchetto F.D., Axon D.J., Sparks W.B., Boksenberg A., 1995b, ApJ 448, 600

Capetti A., Macchetto F., Axon D.J., Sparks W.B., Boksenberg A., 1995c, ApJ 452, L87

Code A.D., et al., 1993, ApJ 403, L63

Condon J.J., Broderick J.J., 1988, AJ 96, 30

Cutri R.M., Rudy R.J., Rieke G.H., Tokunaga A.T., Willner S.P., 1984, ApJ 280, 521

Dahari O., De Robertis M.M., 1988, ApJS 67, 249 
de Vaucouleurs G., de Vaucouleurs A., Corwin H.G., et al., 1991, Third Reference Catalogue of Bright Galaxies. Springer, New York

Edelson R.A., Malkan M.A., 1986, ApJ 308, 59

Edelson R.A., Malkan M.A., Rieke G.H., 1987, ApJ 321, 233

Elvis M., Briel U.G., Henry J.P., 1983, ApJ 268, 105

Evans I.N., Ford H.C., Kinney A.L., et al., 1991, ApJ 369, L27

Evans I.N., Tsvetanov Z., Kriss G.A., 1993, ApJ 417, 82

Fischer J., Geballe T.R., Smith H.A., Simon M., Storey J.V.W., 1987, ApJ 320, 667

Genzel R., Weitzel L., Tacconi-Garman L.E., et al., 1995, ApJ 444, 129

Goodrich R.W., Veilleux S., Hill G.J., 1994, ApJ 422, 521

Haniff C.A., Wilson A.S., Ward M.J., 1988, ApJ 334, 104

Haniff C.A., Ward M.J., Wilson A.S., 1991, ApJ 368, 167

Harrison B., Pedlar A., Unger S.W., et al., 1986, MNRAS 218, 775

Heckman T.M., Balick B., 1983, ApJ 268, 102

Heckman T.M., Blitz L., Wilson A.S., Armus L., Miley G.K., 1989, ApJ 342, 735

Huchra J.P., 1977, ApJS 35, 171

Ichikawa S-i., Okamura S., Kaneko N., Nishimura M., Toyama K., 1987, PASJ 39, 411

Jenkins C.R., 1981, MNRAS 197, 1049

Johnston K.J., Elvis M., Kjer D., Shen P.S.B., 1982, ApJ 262, 61

Kay L., 1994, ApJ 430, 196

Keto E., Ball R., Arens J., Jernigan S., Meixner M., 1992, ApJ 389,223

Kinney A.L., Antonucci R.R.J., Ward M.J., Wilson A.S., Whittle M., 1991, ApJ 377, 100

Kjeldsen H., 1990, special report, Aarhus University

Koski A.T., 1978, ApJ 223, 56

Kotilainen J.K., Prieto M.A., 1995, A\&A 295, 646

Kotilainen J.K., Ward M.J., 1994, MNRAS 266, 953

Kotilainen J.K., Ward M.J., Williger G.M., 1993, MNRAS 263, 655

Koyama K., Inoue H., Tanaka Y., et al., 1989, PASJ 41, 731

Krolik J.H., Begelman M.C., 1986, ApJ 308, L55

Krolik J.H., Lepp S., 1989, ApJ 347, 179

Kukula M.J., Ghosh T., Pedlar A., et al., 1993, MNRAS 264, 893

Lawrence A., Elvis M., 1982, ApJ 256, 410

Macchetto F., Capetti A., Sparks W.B., Axon D.J., Boksenberg A., 1994, ApJ 435, L15

Maiolino R., et al., 1995, ApJ 446, 561

Mauder W., Weigelt G., Appenzeller I., Wagner S.J., 1994, A\&A 285, 44

Meixner M., Puchalsky R., Blitz L., Wright M., Heckman T., 1990, ApJ 354, 158

Miley G.K., Neugebauer G., Soifer B.T., 1985, ApJ 293, L11

Miller J.S., Goodrich R.W., 1990, ApJ 355, 456

Miller J.S., Goodrich R.W., Mathews W.G., 1991, ApJ 378, 47

Mulchaey J.S., et al., 1994, ApJ 436, 586

Mundell C.G, Pedlar A., Axon D.J., Meaburn J., Unger S.W., 1995, MNRAS 277, 641

Nakajima T., et al., 1991, ApJ 373, 452
Neff S.G., de Bruyn A.G., 1983, A\&A 128, 318

Pedlar A., Unger S.W., Booler R.V., 1984, MNRAS 207, 193

Pedlar A., Meaburn J., Axon D.J., et al., 1989, MNRAS 238, 863

Pedlar A., et al., 1993, MNRAS 263, 471

Perez E., Gonzalez-Delgado R., Tadhunter C., Tsvetanov Z., 1989, MNRAS 241, 31P

Planesas P., Scoville N.Z., Myers S.T., 1991, ApJ 369, 364

Pogge R.W., 1989, ApJ 345, 730

Pogge R.W., De Robertis M.M., 1993, ApJ 404, 563

Pogge R.W., De Robertis M.M., 1995, ApJ 451, 585

Robinson A., Vila-Vilaro B., Axon D.J., et al., 1994, A\&A 291, 351

Roche P.F., Aitken D.K., Smith C.H., Ward M.J., 1991, MNRAS 248, 606

Rodriguez-Espinosa J.M., Rudy R.J., Jones B., 1987, ApJ 312, 555

Ruiz M., Rieke G.H., Schmidt G.D., 1994, ApJ 423, 608

Salamanca I., et al., 1994, A\&A 282, 742

Schild R., Tresch-Fienberg R., Huchra J., 1985, AJ 90, 441

Schmidt G.D., Miller J.S., 1985, ApJ 290, 517

Simkin S.M., van Gorkom J., Hibbard J., Su H.-J., 1987, Sci 235,1367

Tadhunter C., Tsvetanov Z., 1989, Nat 341, 422

Taniguchi Y., Kameya O., Nakai N., Kawara K., 1990, ApJ 358,132

Tran H.D., Miller J.S., Kay L.E., 1992, ApJ 397, 452

Tresch-Fienberg R., Fazio G.G., Gezari D.Y., et al., 1987, ApJ 312,542

Truong K.Q., Bruhweiler F.C., 1991, ApJ 379, 609

Tsvetanov Z., Walsh J.R., 1992, ApJ 386, 485

Tsvetanov Z., Kotilainen J.K., Ward M.J., 1996 (in preparation)

Ulvestad J.S., Wilson A.S., 1984, ApJ 278, 544

Ulvestad J.S., Wilson A.S., 1989, ApJ 343, 659

Ulvestad J.S., Wilson A.S., Sramek R.A., 1981, ApJ 247, 419

Unger S.W., Pedlar A., Axon D.J., et al., 1987, MNRAS 228, 671

Unger S.W., Lewis J.R., Pedlar A., Axon D.J., 1992, MNRAS 258,371

Vila-Vilaro B., et al., 1995, A\&A 302, 58

Wagner S., 1987, A\&A 185, 77

Ward M.J., Elvis M., Fabbiano G., et al., 1987, ApJ 315, 74

Warwick R.S., Koyama K., Inoue H., et al., 1989, PASJ 41, 739

Whittle M., Pedlar A., Meurs E.J.A., et al., 1988, ApJ 326, 125

Wilson A.S., 1988, A\&A 206, 41

Wilson A.S., Ulvestad J.S., 1983, ApJ 275, 8

Wilson A.S., Ulvestad J.S., 1987, ApJ 319, 105

Wilson A.S., Tsvetanov Z., 1994, AJ 107, 1227

Wilson A.S., Baldwin J.S., Sun S.-D., Wright A.E., 1986, ApJ 310, 121

Wilson A.S., Ward M.J., Haniff C.A., 1988, ApJ 334, 121

Wilson A.S., Helfer T.T., Haniff C.A., Ward M.J., 1991, ApJ 381,79

Wilson A.S., Elvis M., Lawrence A., Bland-Hawthorn J., 1992, ApJ 391, L75 


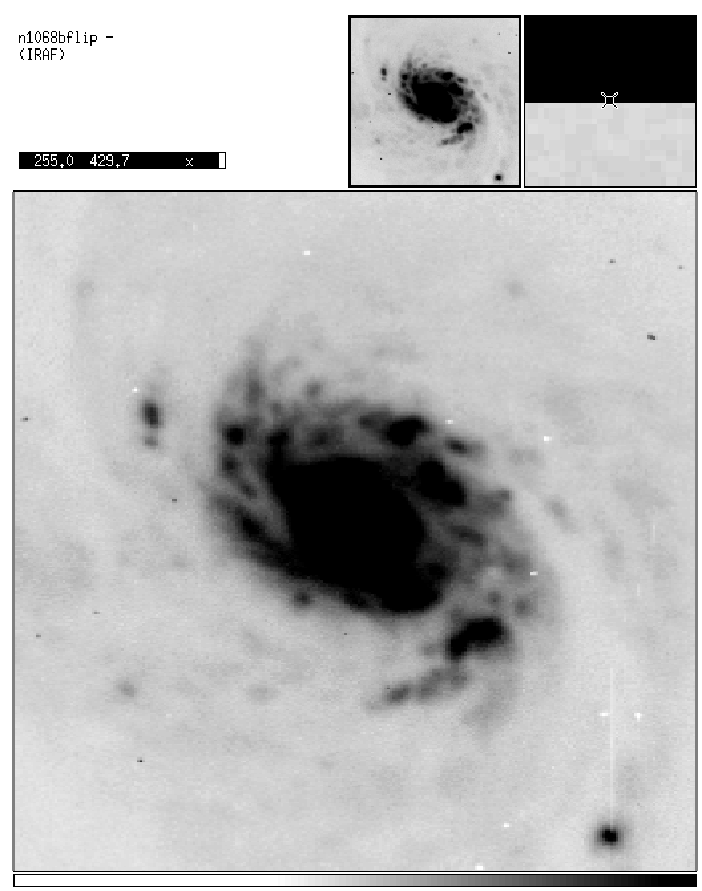

Plate 1. The $B$ band image of NGC 1068. The size of the image is $70 \times 70^{\prime \prime}(7.7 \times 7.7 \mathrm{kpc})$. Knots $2-4$ are marked on the map. In this and other plates, north is up, east to the left and the nucleus of the galaxy in the exact center of the plate

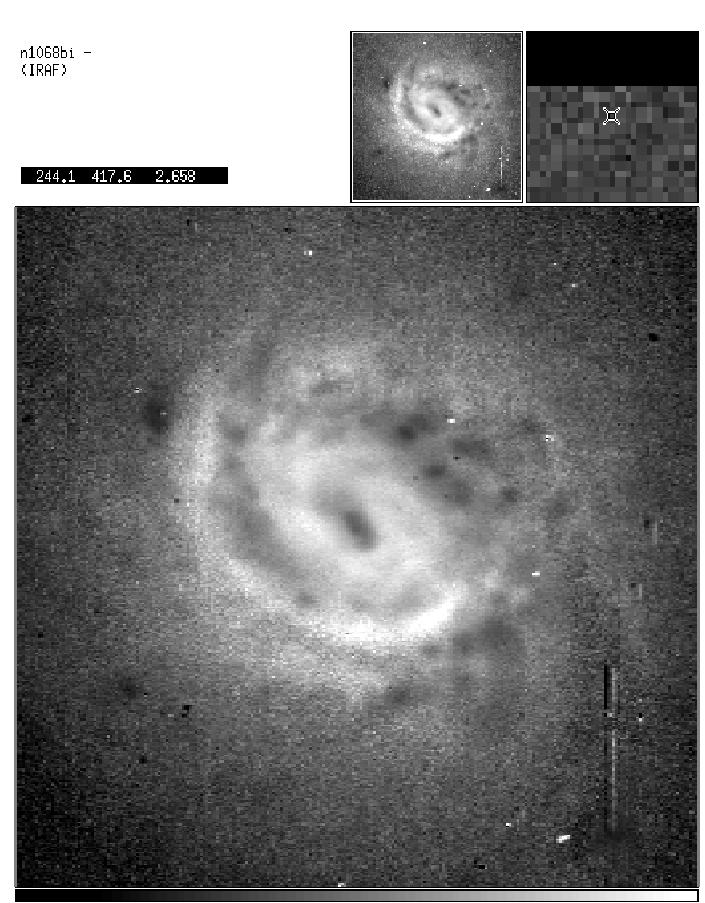

Plate 2. The $B-I$ map of NGC 1068. The size of the image is $70 \times 70^{\prime \prime}(7.7 \times 7.7 \mathrm{kpc})$. In this and other colour maps, dark shades indicate blue and light shades red emission. The $B-I$ colour coding is from 1.1 to 3.8

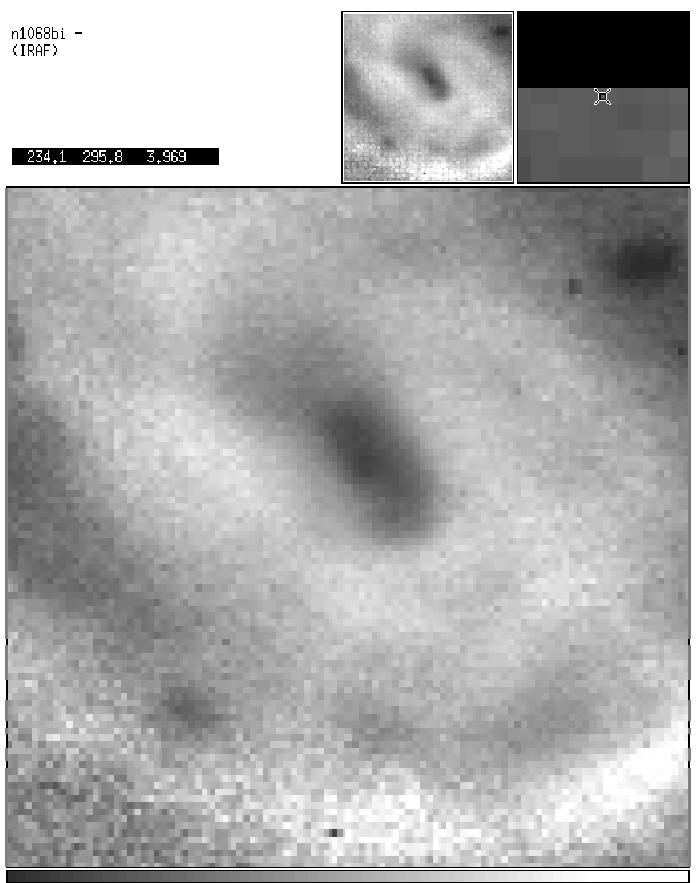

Plate 3. The $B-I$ map of the innermost $20 \times 20^{\prime \prime}(2.2 \times 2.2 \mathrm{kpc})$ of NGC 1068, showing the blue elongation parallel to the radio jet axis. The $B-I$ colour coding is from 1.4 to 2.7

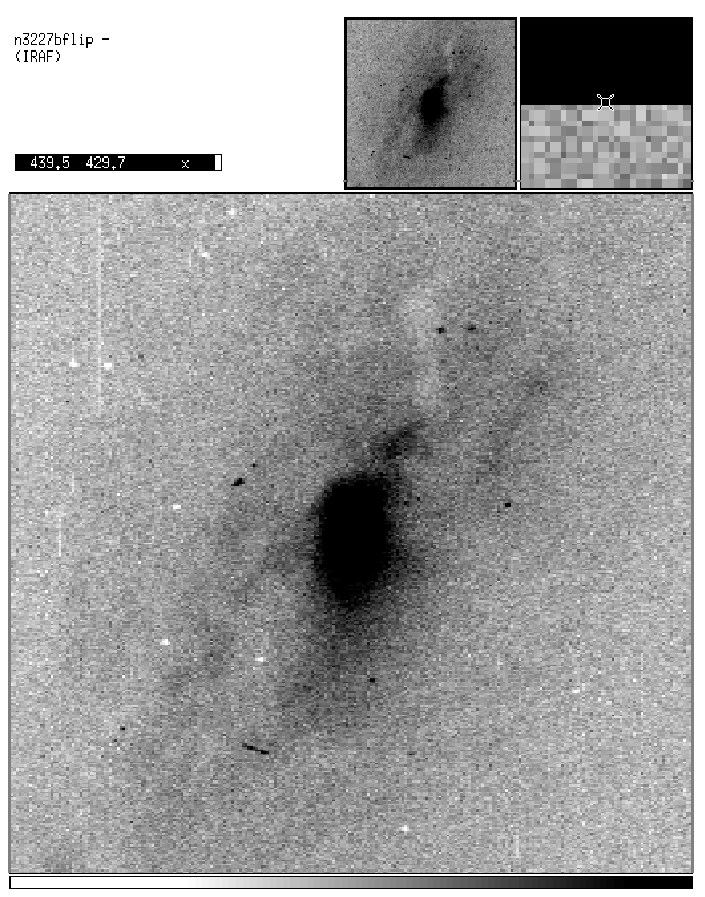

Plate 4. The $B$ band image of NGC 3227. The scale of the full image is $70 \times 70^{\prime \prime}(7 \times 7 \mathrm{kpc})$ 


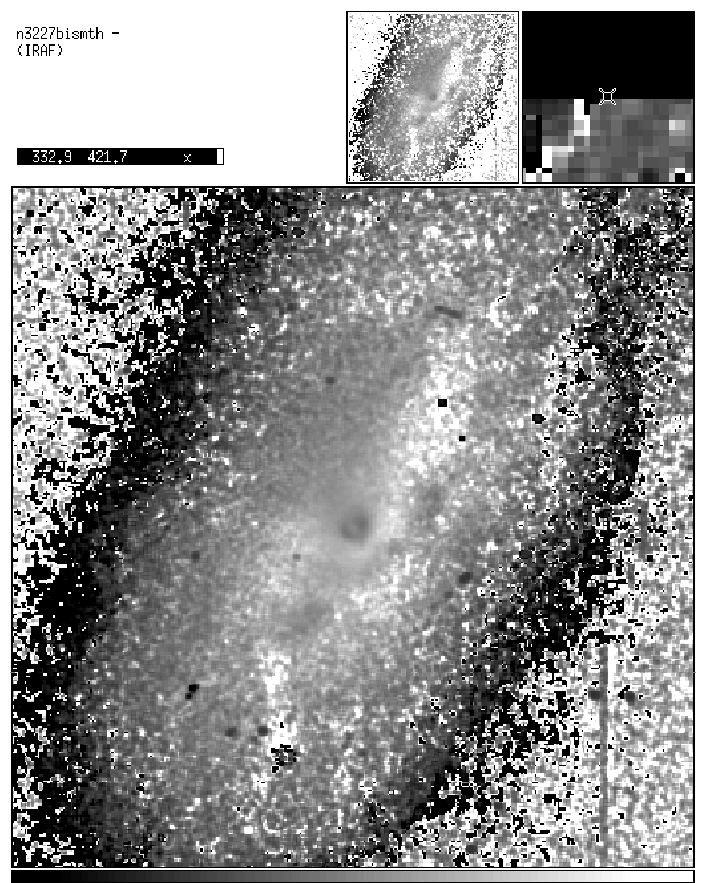

Plate 5. The $B-I$ map of NGC 3227 . The scale of the full image is $70 \times 70^{\prime \prime}(7 \times 7 \mathrm{kpc})$. The $B-I$ colour coding is from 0.50 to 5.3 . Note the complex structure

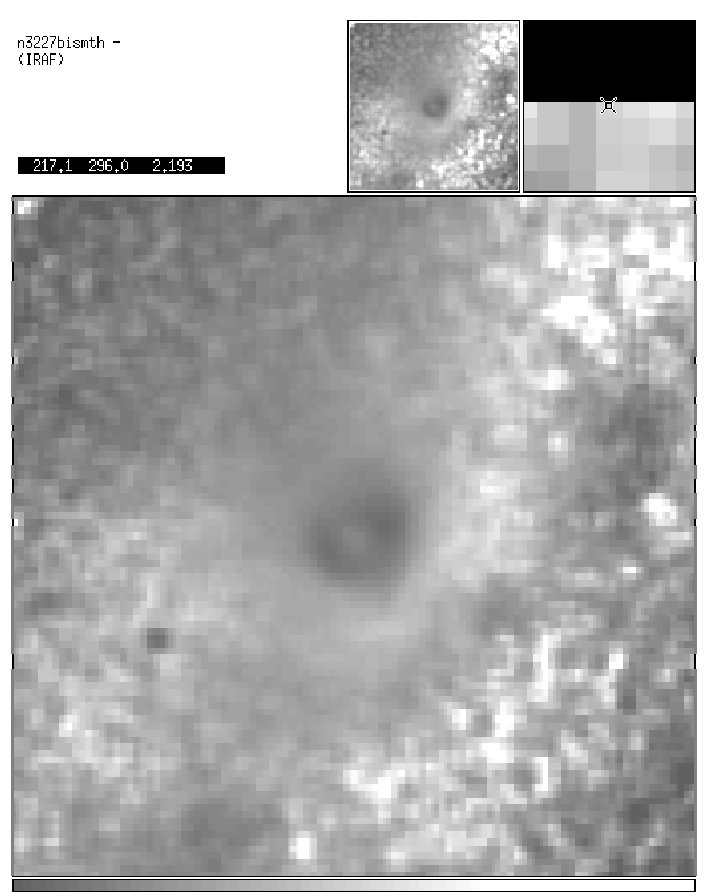

Plate 6. The $B-I$ map of the innermost $20 \times 20^{\prime \prime}(2 \times 2 \mathrm{kpc})$ of NGC 3227 . The $B-I$ colour coding is from 0.87 to 4.6 . Note the two blue maxima straddling the nucleus

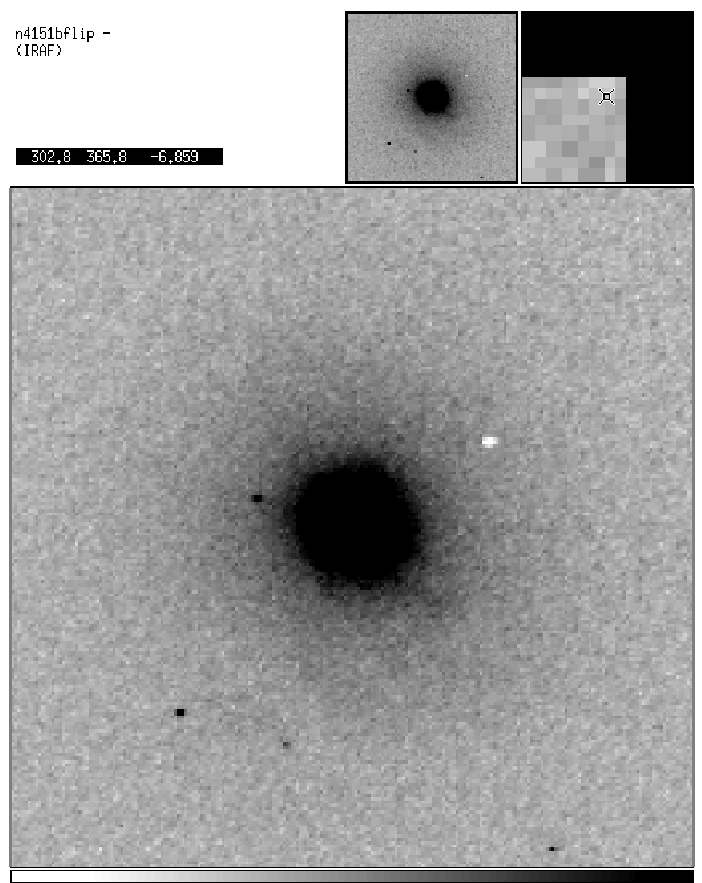

Plate 7. The $B$ band image of NGC 4151 . The size of the image is $40 \times 40^{\prime \prime}=4 \times 4 \mathrm{kpc}$

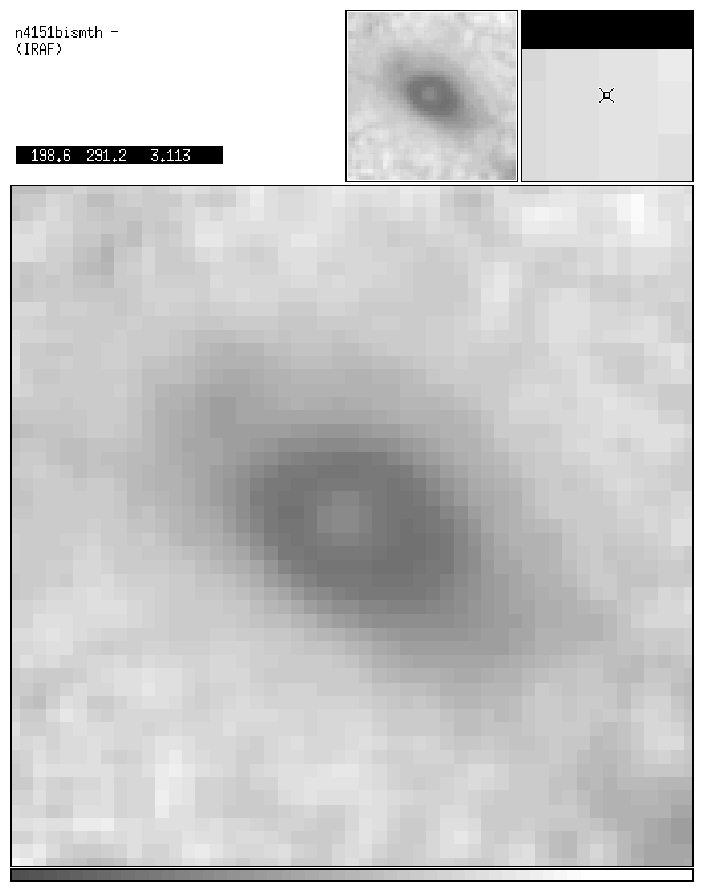

Plate 8. The $B-I$ map of the innermost $10 \times 10^{\prime \prime}(1 \times 1 \mathrm{kpc})$ of NGC 4151. The $B-I$ colour coding is from 1.9 to 3.3. Note the blue elongation parallel to the radio jet axis and two blue maxima on either side of the nucleus 


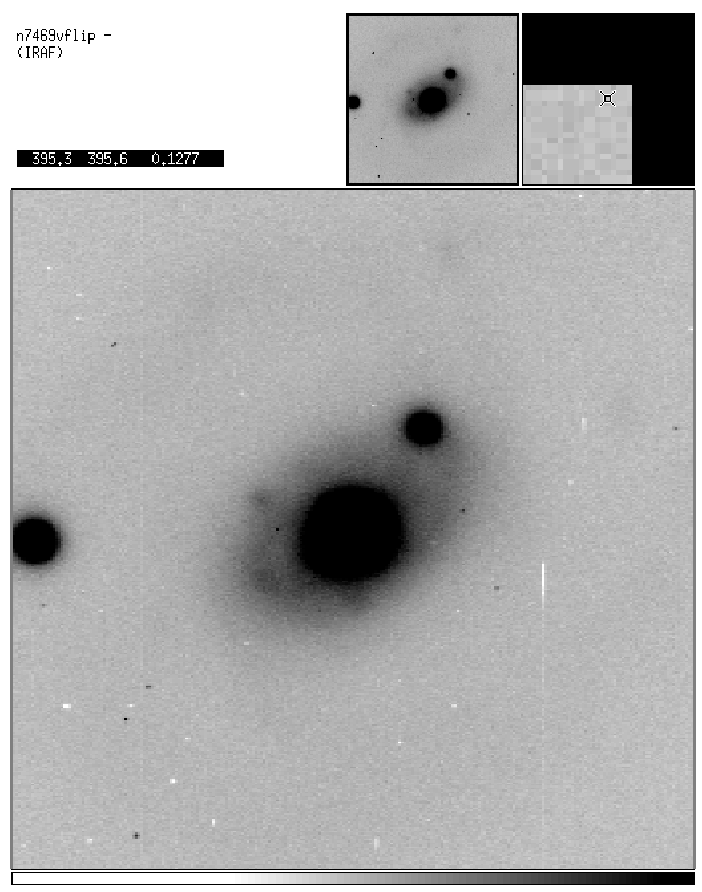

Plate 9. The $V$ band image of NGC 7469. The size of the image is $70 \times 70^{\prime \prime}=34 \times 34 \mathrm{kpc}$

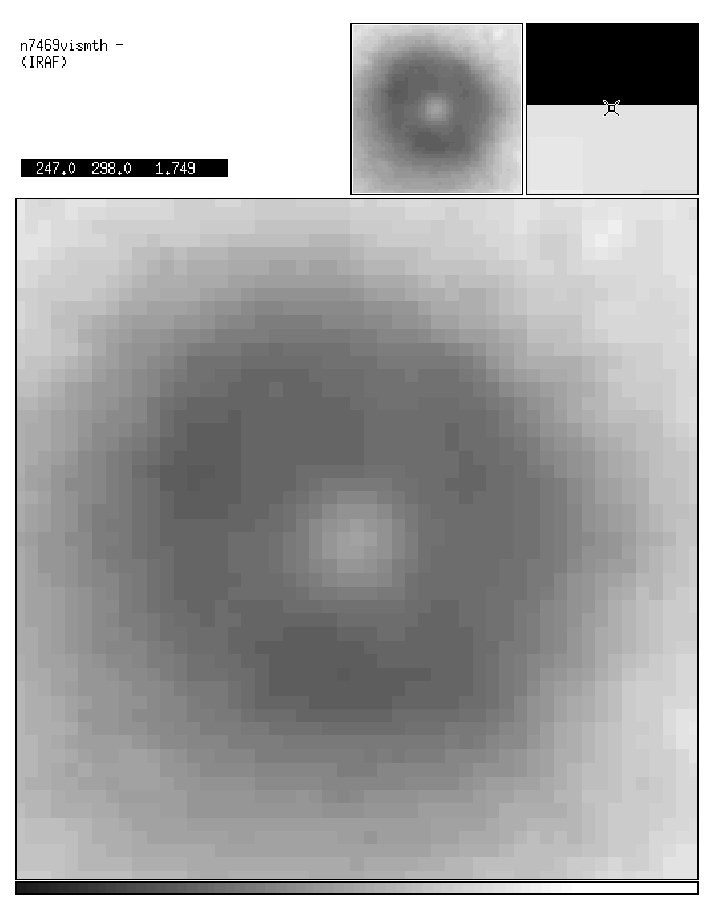

Plate 10. The $V-I$ map of the innermost $10 \times 10^{\prime \prime}(4.9 \times$ $4.9 \mathrm{kpc}$ ) of NGC 7469 . The $V-I$ colour coding is from 1.1 to 1.6. Note the circumnuclear ring with a diameter of $3^{\prime \prime}$

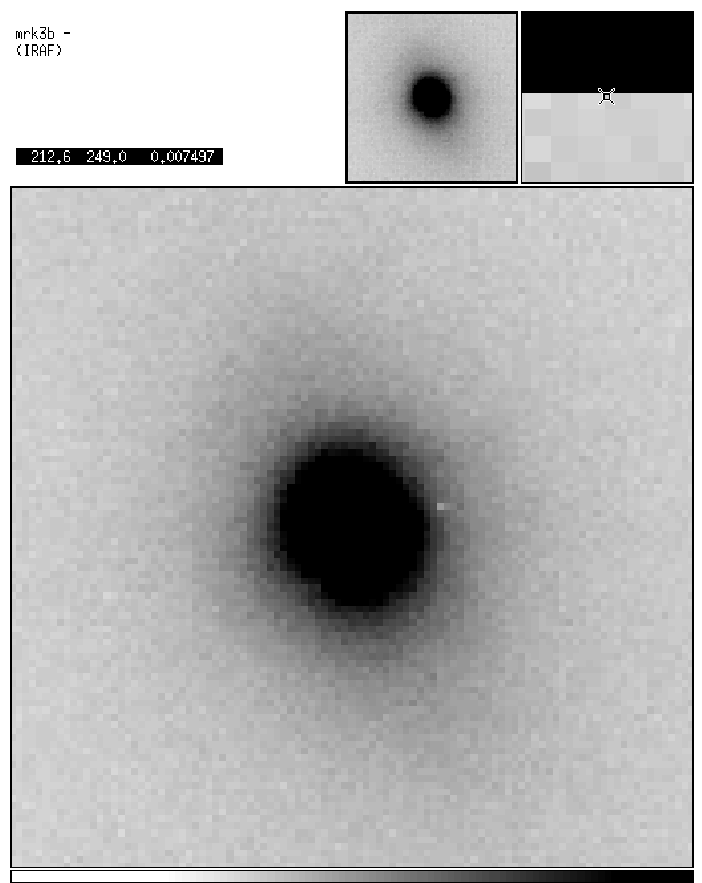

Plate 11. The $B$ band image of Mrk 3. The size of the image is $20 \times 20^{\prime \prime}=8.2 \times 8.2 \mathrm{kpc}$

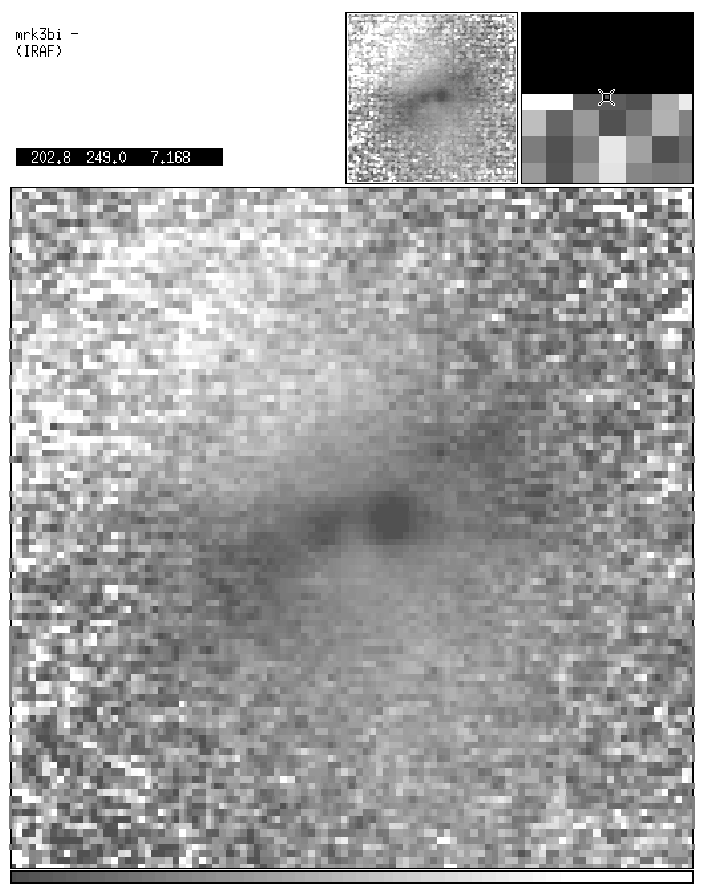

Plate 12. The $B-I$ map of the innermost $20 \times 20^{\prime \prime}(8.2 \times$ $8.2 \mathrm{kpc}$ ) of Mrk 3. The $B-I$ colour coding is from 1.8 to 4.2 . Note the elongated blue region with two maxima 


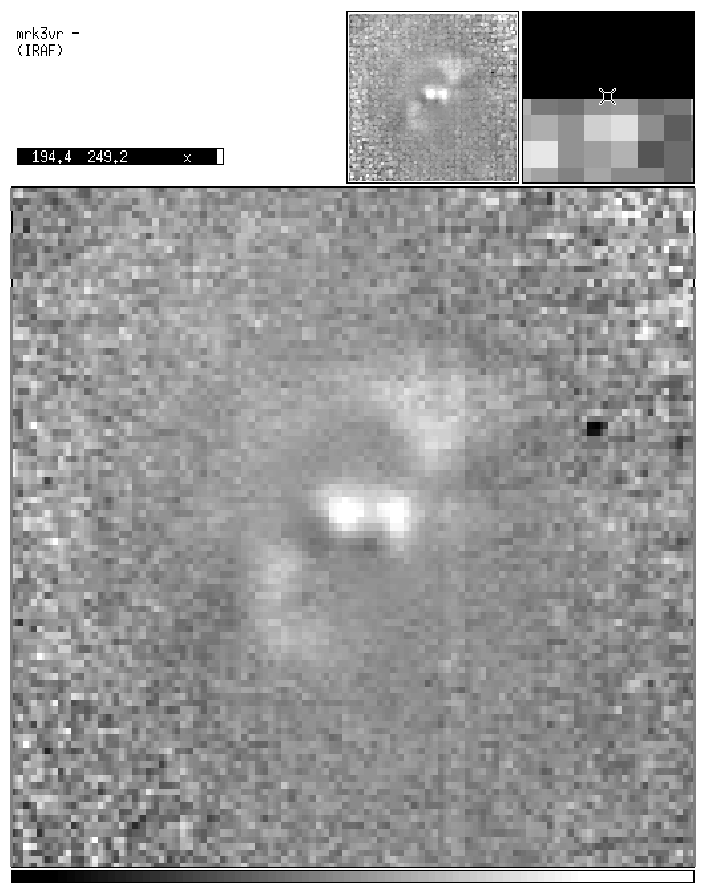

Plate 13. The $V-R$ "excitation" map of the innermost $20 \times$ $20^{\prime \prime}(8.2 \times 8.2 \mathrm{kpc})$ of Mrk 3 . The $V-R$ colour coding is from 0.38 to 1.1 . Note the tightly wound spiral structure and the two red maxima

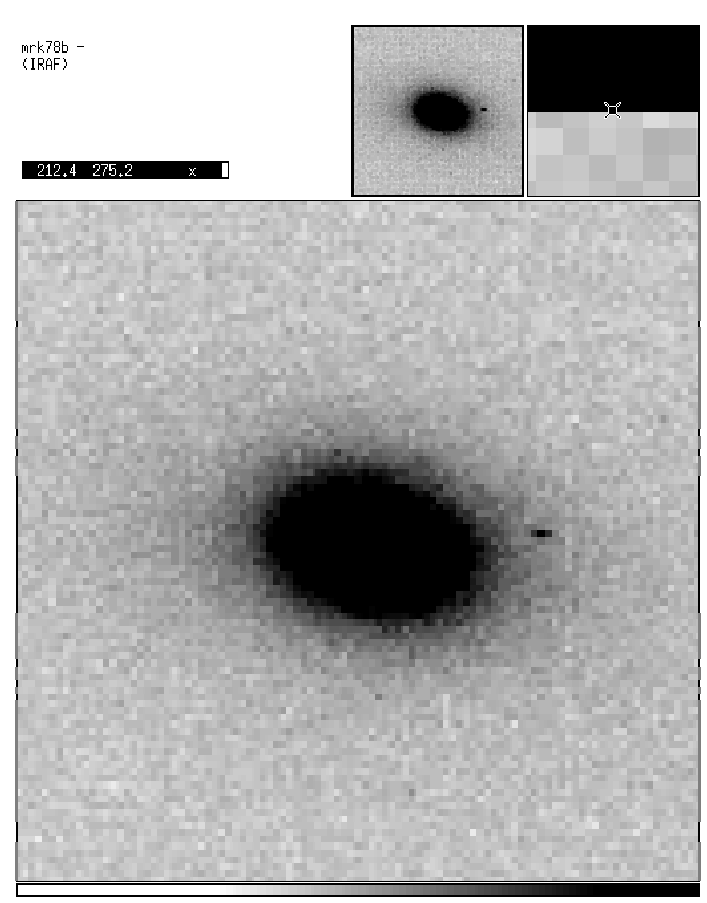

Plate 14. The $B$ band image of Mrk 78. The size of the image is $20 \times 20^{\prime \prime}=23 \times 23 \mathrm{kpc}$

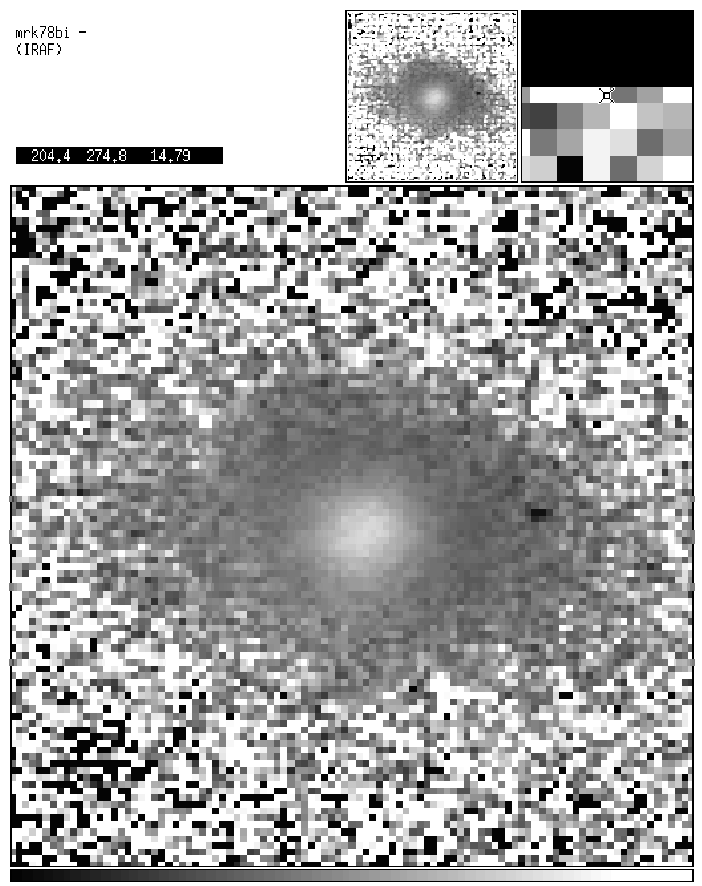

Plate 15. The $B-I$ map of the innermost $20 \times 20^{\prime \prime}(23 \times 23 \mathrm{kpc})$ of Mrk 78. The $B-I$ colour coding is from 1.2 to 4.0 . Note the blue arclike structure $\mathrm{N}$ of the nucleus

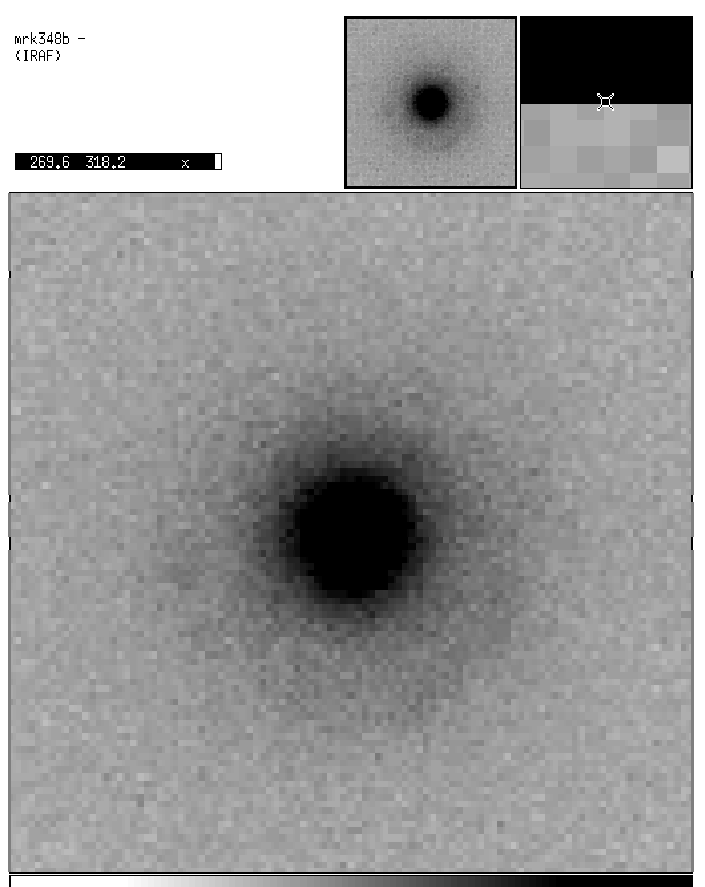

Plate 16. The $B$ band image of Mrk 348. The size of the image is $20 \times 20^{\prime \prime}=8.4 \times 8.4 \mathrm{kpc}$ 


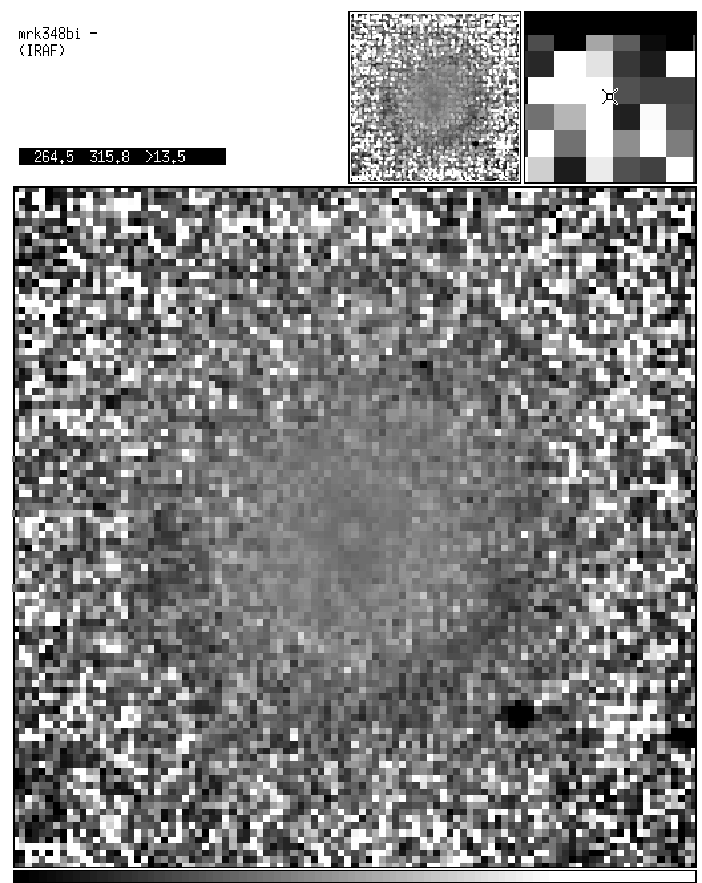

Plate 17. The $B-I$ map of the innermost $20 \times 20^{\prime \prime}(8.4 \times$ $8.4 \mathrm{kpc}$ ) of Mrk 348. The $B-I$ colour coding is from 0.19 to 4.0

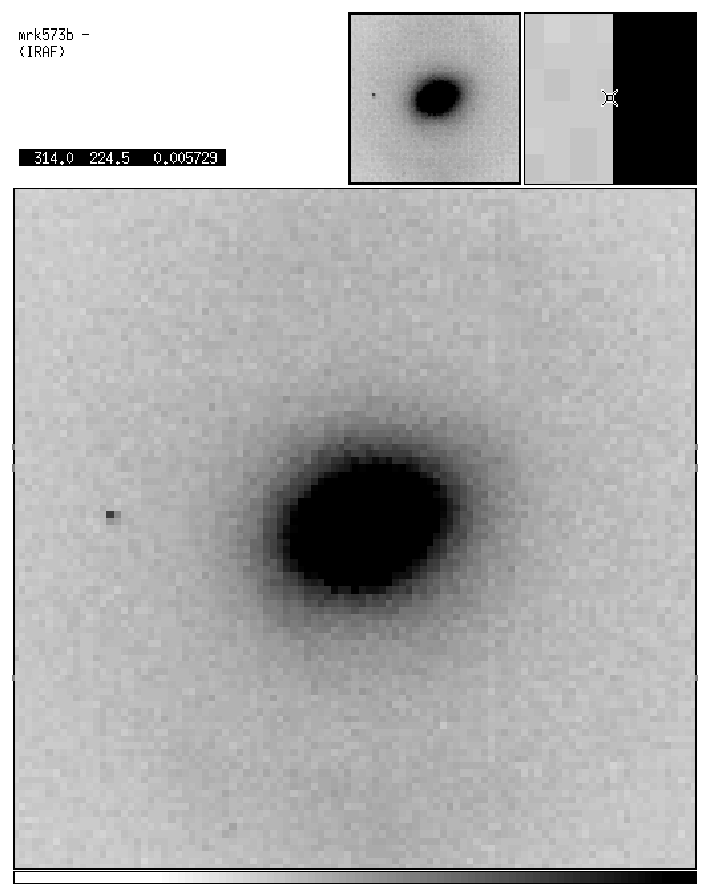

Plate 18. The $B$ band image of Mrk 573. The size of the image $20 \times 20^{\prime \prime}=10.2 \times 10.2 \mathrm{kpc}$

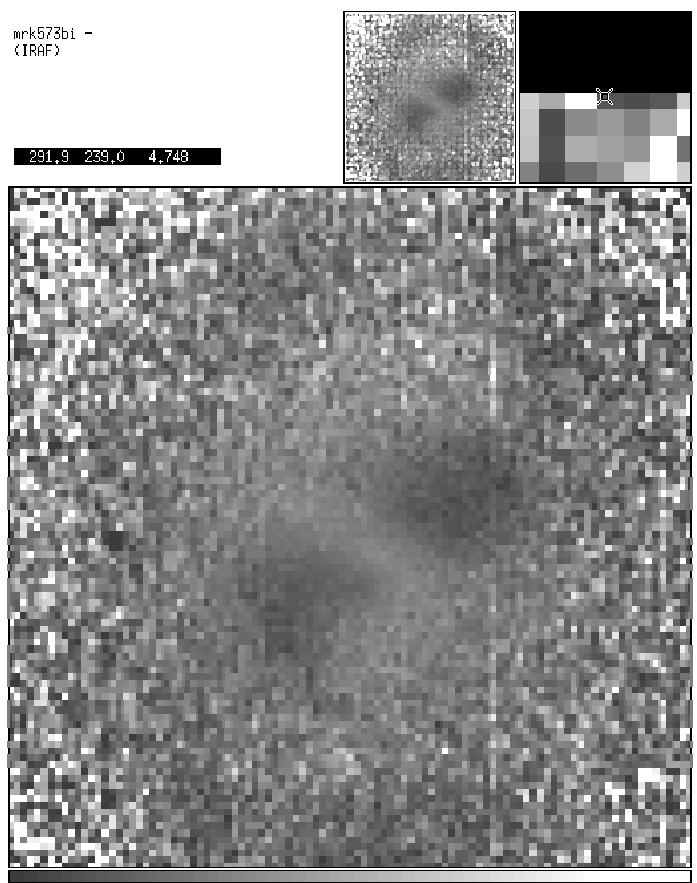

Plate 19. The $B-I$ map of the innermost $20 \times 20^{\prime \prime}(10.2 \times$ $10.2 \mathrm{kpc}$ ) of Mrk 573. The $B-I$ colour coding is from 0.14 to 4.2. Note the blue double structure and the red bridge perpendicular to them. There is also evidence for a faint blue spiral structure further away from the nucleus

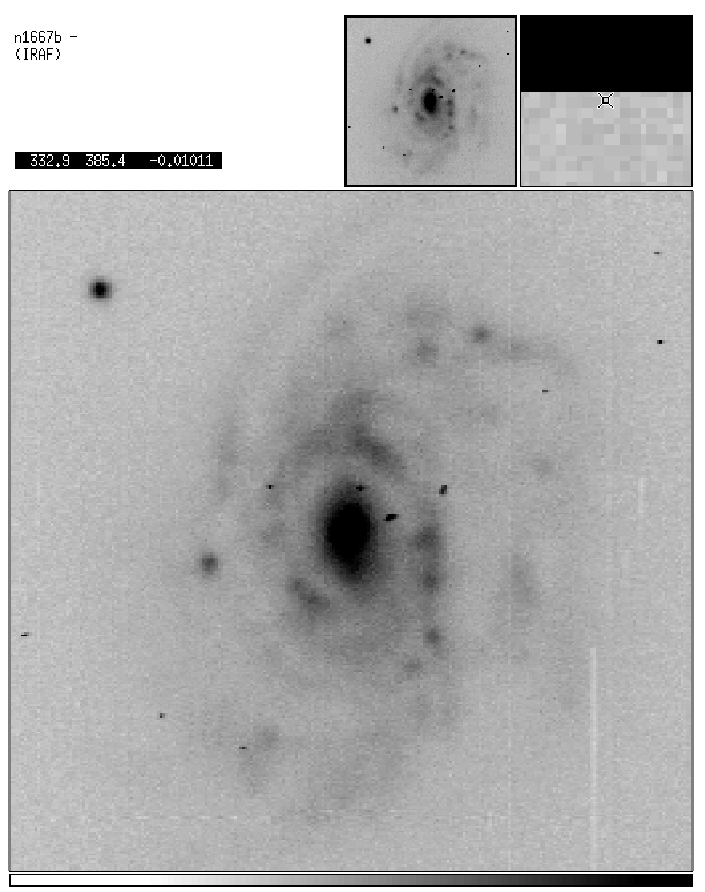

Plate 20. The $B$ band image of NGC 1667. The size of the image is $60 \times 60^{\prime \prime}(28 \times 28 \mathrm{kpc})$ 


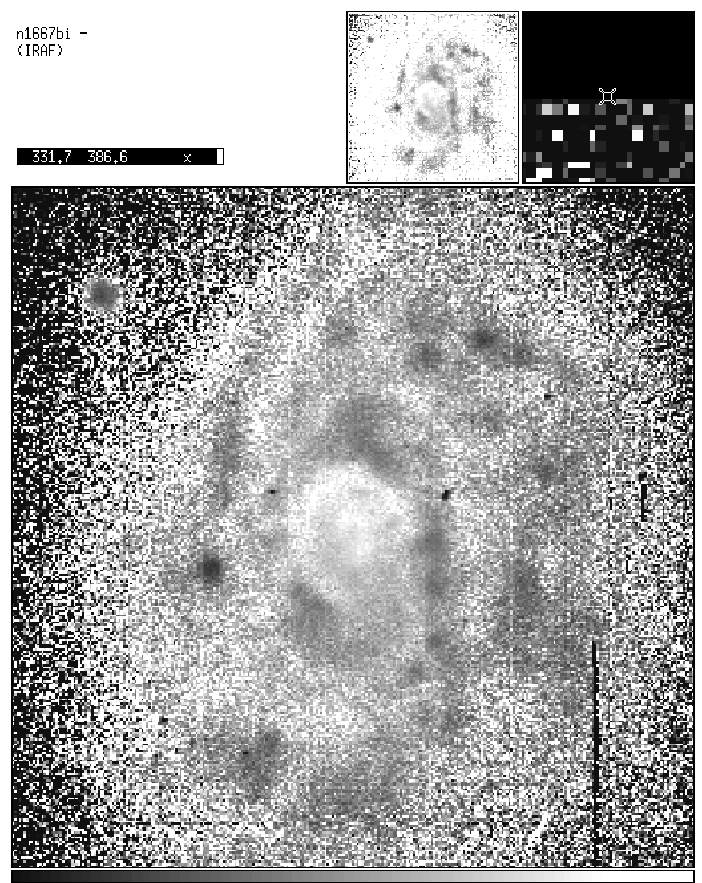

Plate 21. The $B-I$ map of the innermost $60 \times 60^{\prime \prime}(28 \times 28 \mathrm{kpc})$ of NGC 1667. The $B-I$ colour coding is from 0.47 to 3.4 . The H II regions in the spiral arms and the dust geometry are clearly resolved

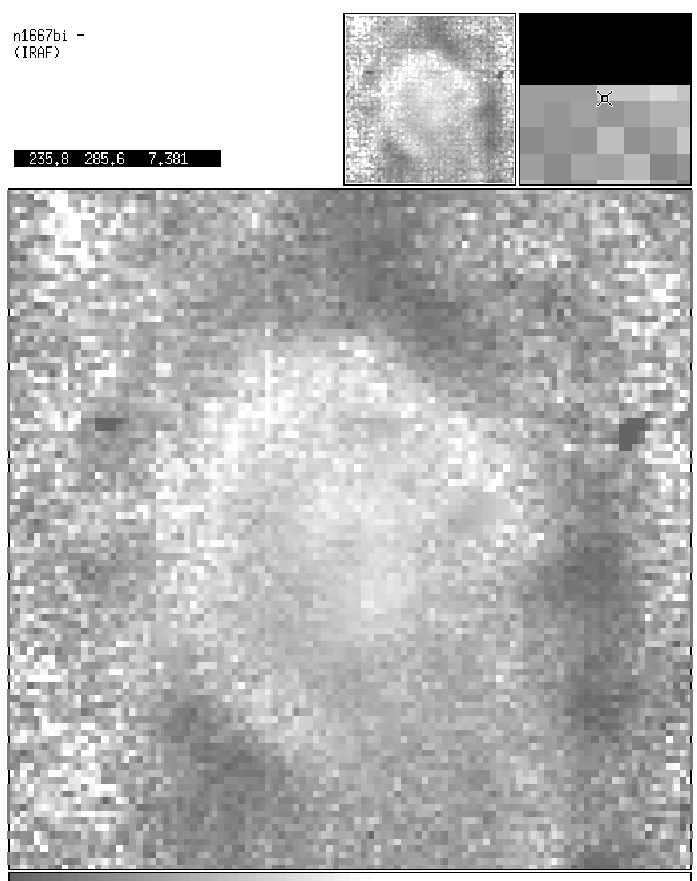

Plate 22. The $B-I$ map of the innermost $20 \times 20^{\prime \prime}(9.4 \times$ $9.4 \mathrm{kpc}$ ) of NGC 1667. The $B-I$ colour coding is from 0.56 to 3.4. Note the double structure of red material across the nucleus 